\title{
Origin of the Soft Excess in X\#Ray Pulsars
}

\section{Citation}

Hickox, Ryan C., Ramesh Narayan, and Timothy R. Kallman. 2004. "Origin of the Soft Excess in X\#Ray Pulsars." The Astrophysical Journal 614 (2): 881-96. https://doi.org/10.1086/423928.

\section{Permanent link}

http://nrs.harvard.edu/urn-3:HUL.InstRepos:41384959

\section{Terms of Use}

This article was downloaded from Harvard University's DASH repository, and is made available under the terms and conditions applicable to Other Posted Material, as set forth at http:// nrs.harvard.edu/urn-3:HUL.InstRepos:dash.current.terms-of-use\#LAA

\section{Share Your Story}

The Harvard community has made this article openly available.

Please share how this access benefits you. Submit a story.

Accessibility 


\title{
ORIGIN OF THE SOFT EXCESS IN X-RAY PULSARS
}

\author{
Ryan C. Hickox and Ramesh Narayan \\ Harvard-Smithsonian Center for Astrophysics, 60 Garden Street, Cambridge, MA 02138 \\ AND \\ Timothy R. KaLlman \\ NASA Goddard Space Flight Center, Laboratory for High Energy Astrophysics, Code 665, Greenbelt, MD 20771 \\ Received 2004 April 8; accepted 2004 July 3
}

\begin{abstract}
The spectra of many X-ray pulsars show, in addition to a power law, a low-energy component that has often been modeled as a blackbody with $k T_{\mathrm{BB}} \sim 0.1 \mathrm{keV}$. However, the physical origin of this soft excess has remained a mystery. We examine a sample of well-studied bright X-ray pulsars, which have been observed using ROSAT, ASCA, Ginga, RXTE, BeppoSAX, Chandra, and XMM-Newton. In particular, we consider the Magellanic Cloud pulsars SMC X-1, LMC X-4, XTE J0111.2-7317, and RX J0059.2-7138 and the Galactic sources Her X-1, 4U 1626-67, Cen X-3, and Vela X-1. We show that the soft excess is a very common if not ubiquitous feature intrinsic to X-ray pulsars. We evaluate several possible mechanisms for the soft emission, using theoretical arguments, as well as observational clues, such as spectral shapes, eclipses, pulsations of the soft component, and superorbital modulation of the source flux. We find that reprocessing of hard X-rays from the neutron star by the inner region of the accretion disk is the only process that can explain the soft excess in all the pulsars with $L_{\mathrm{X}} \gtrsim 10^{38} \mathrm{ergs} \mathrm{s}^{-1}$. Other mechanisms, such as emission from diffuse gas in the system, are important in less luminous objects.
\end{abstract}

Subject headings: accretion, accretion disks — pulsars: general — stars: neutron — X-rays: binaries

\section{INTRODUCTION}

X-ray binary pulsars (XBPs) have provided a fruitful laboratory for high-energy astrophysics since their discovery in the 1970s (for a review, see Nagase 1989). Many XBPs have been observed extensively and are useful for studying a variety of astrophysical processes, including accretion onto compact objects, binary interactions, and the effects of strong magnetic fields.

In recent years, studies of XBPs with orbiting observatories such as ROSAT, ASCA, Ginga, RXTE, BeppoSAX, Chandra, and $X M M$-Newton have allowed for closer scrutiny of the relevant physics. In particular, most bright XBPs outside the Galaxy (for which soft X-rays are not heavily absorbed by Galactic gas) have shown a marked soft excess in their X-ray spectra above the standard power-law model (Nagase 2002). Authors have proposed a variety of simple models to describe this soft excess, but there has been considerable debate over the origin of this feature in a number of sources. For example, the soft component in LMC X-4 has been modeled as a thermal bremsstrahlung-shaped fan beam (Woo et al. 1996), powerlaw emission from the upper accretion column (Paul et al. 2002), diffuse bremsstrahlung emission, and/or reprocessing by accreting material at the magnetosphere ( $\mathrm{La}$ Barbera et al. 2001). In this paper we seek to narrow these candidate mechanisms for the soft excess, by examining the physical processes in light of a wide range of observations.

The Galactic XBP Her X-1 has a well-studied soft excess that can be explained by reprocessing of hard X-rays by the inner edge of the accretion disk (Endo et al. 2000; Ramsay et al. 2002). At the moment Her X-1 is the XBP for which the soft excess is best observed and understood, and we use it to guide our study of other sources.

Because the Galaxy's disk contains X-ray-absorbing gas, the soft excess is not easy to observe in most Galactic XBPs. This emission is best seen in the Magellenic Clouds, at high
Galactic latitude, where there is not much low-energy absorption. The bright XBPs in these nearby galaxies are generally more luminous than those in the Milky Way and are close enough to obtain good spectra with a high signal-tonoise ratio. In a survey of $A S C A$ observations of the SMC, Yokogawa et al. (2003) found $30 \mathrm{XBPs}$, of which three have $\mathrm{X}$-ray luminosities large enough to extract good spectra. All three of these sources (SMC X-1, RX J0059.2-7138, and XTE J0111.2-7317) show a clear soft excess feature in the spectrum. The one comparably bright XBP in the LMC (LMC X-4) also shows a soft excess. All four of these sources have $L_{\mathrm{X}} \sim 10^{38} \mathrm{ergs} \mathrm{s}^{-1}$.

$4 \mathrm{U} 1626-67$, Cen X-3 and Vela X-1 in the Galaxy, and EXO 053109-6609.2 and A0538-66 in the LMC also show soft excess features (Angelini et al. 1995; Burderi et al. 2000; Haberl 1994; Haberl et al. 2003; Mavromatakis \& Haberl 1993). In addition, a recent survey of the SMC with $X M M$ has revealed soft components in several lower luminosity pulsars (Sasaki et al. 2003). In $\S 2$ we summarize observational studies of the XBPs mentioned above, and in $\S 3$ we examine how common the soft excess feature is in the total population of XBPs and introduce possible mechanisms for its origin. In $\S \S 4-7$ we evaluate these possibilities in terms of both theory and observations. In $\S 8$ we discuss the implications of the results and future work, and in $\S 9$ we summarize our results.

\section{OBSERVATIONAL BACKGROUND}

Here we review a number of observations of XBPs with a soft excess. Orbital and spectral parameters for each source are compiled in Table 1.

\subsection{Her $X-1$}

Her X-1 was one of the first known X-ray pulsars (Tananbaum et al. 1972) and is one of the few with a low-mass companion. It has a pulse period of $1.24 \mathrm{~s}$ and an orbital period of 1.7 days. The 
TABLE 1

Orbital and Spectral Parameters for XBPs with Soft Excesses

\begin{tabular}{|c|c|c|c|c|c|c|c|c|}
\hline Parameter & Her $X-1^{a}$ & SMC X-1 ${ }^{\mathrm{b}}$ & $\mathrm{LMC} \mathrm{X}-4^{\mathrm{c}}$ & $\begin{array}{c}\text { XTE } \\
\text { J0111.2-7317 }\end{array}$ & $\begin{array}{c}\mathrm{RX} \\
\mathrm{J} 0059.2-7138^{\mathrm{e}}\end{array}$ & $4 \mathrm{U} 1626-67^{\mathrm{f}}$ & Cen $X-3^{g}$ & Vela $X-1^{\mathrm{h}}$ \\
\hline Location & Galaxy & SMC & LMC & SMC & SMC & Galaxy & Galaxy & Galaxy \\
\hline 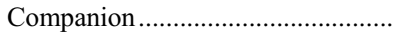 & A star & B0 & O7 III-O IV & $\mathrm{Be}$ & $\mathrm{Be}$ & Low-mass & $\mathrm{O} 6-\mathrm{O} 8$ & B0.5Ib \\
\hline Distance $(\mathrm{kpc})$ & $\sim 5$ & 65 & 50 & 65 & 65 & Unknown & $\sim 8$ & 1.9 \\
\hline 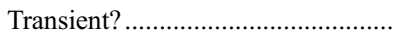 & No & No & No & Yes & Yes & No & No & No \\
\hline$P_{\text {orb }}$ (days) & 1.7 & 3.9 & 1.4 & & . & 0.03 & 2.1 & 8.96 \\
\hline$P_{\text {pulse }}(\mathrm{s})$ & 1.24 & 0.7 & 13.5 & 30.95 & 2.76 & 7.7 & 4.8 & 283 \\
\hline$P_{\text {superorbital }}$ (days) $\ldots \ldots \ldots \ldots \ldots \ldots$ & 35 & $40-60$ & 30 & $\ldots$ & $\ldots$ & $\ldots$ & & $\ldots$ \\
\hline$L_{\mathrm{X}}\left(\mathrm{ergs} \mathrm{s}^{-1}\right)$ & $2 \times 10^{37}$ & $2.4 \times 10^{38}$ & $1.2 \times 10^{38}$ & $1.8 \times 10^{38}$ & $2.6 \times 10^{38}$ & $7.7 \times 10^{34} D_{\mathrm{kpc}}^{2}$ & $\sim 10^{38}$ & $\sim 10^{36}$ \\
\hline 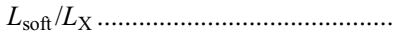 & 0.04 & 0.036 & 0.064 & $\sim 0.10$ & $\sim 0.35$ & $\sim 0.03$ & $\sim 0.5$ & $\sim 0.01$ \\
\hline Soft pulses? & Yes & Yes & Yes & Yes & No & No & Yes & No \\
\hline 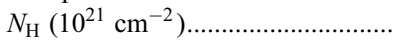 & $0.05, \sim 100^{\mathrm{i}}$ & $2-5$ & $\sim 0.5$ & $1.8_{-0.2}^{+0.3}$ & $(0.42-0.50) \pm 0.05$ & $1.1 \pm 0.2$ & $19.5 \pm 0.3$ & $4.2 \pm 0.5,150^{\mathrm{i}}$ \\
\hline$\Gamma_{\text {hard }}$ & $0.9-1.2$ & $\sim 0.9$ & $0.5-0.7$ & $0.76_{-0.02}^{+0.01}$ & $0.43 \pm 0.05$ & $0.83 \pm 0.01$ & $1.208 \pm 0.007$ & 1.4 \\
\hline$E_{\text {cut }}(\mathrm{keV})$ & 24 & $\sim 6$ & $16-18$ & $\ldots$ & $6.4_{-0.9}^{+0.7}$ & $29 \pm 2$ & $13.79 \pm 0.13$ & $\ldots$ \\
\hline 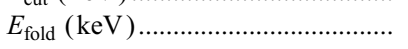 & 15 & $15-35$ & $18-35$ & $\ldots$ & $9.3_{-4.3}^{+11.2}$ & $9_{-1}^{+2}$ & $8.39 \pm 0.19$ & $\ldots$ \\
\hline$E_{\mathrm{Fe}}(\mathrm{keV})$ & $\sim 6.5$ & $\sim 6.5$ & $\sim 6.4$ & 6.4 & $\ldots$ & $\ldots$ & $6.66 \pm 0.03$ & 6.4 \\
\hline$E_{\text {cyc }}(\mathrm{keV}) \ldots$ & $42.1 \pm 0.3$ & $\ldots$ & $100_{-15}^{+80}$ & $\ldots$ & $\ldots$ & $33 \pm 1$ & $30.6 \pm 0.6$ & 24,55 \\
\hline Soft excess model types ${ }^{\mathrm{j}} \ldots \ldots \ldots \ldots \ldots \ldots$ & $\mathrm{BB}, \mathrm{BB}+\mathrm{LE}$ & $\mathrm{BB}, \mathrm{TB}, \mathrm{SPL}$ & $\mathrm{BB}, \mathrm{BB}+\mathrm{TB}, \mathrm{COM}, \mathrm{SPL}$ & SPL & MEK, SPL & BB & BB & $\mathrm{TB}$, lines \\
\hline$k T_{\mathrm{BB}}(\mathrm{keV})$ & $0.09-0.12$ & $0.15-0.18$ & 0.15 & $\ldots$ & $\ldots$ & $\ldots$ & $\ldots$ & $\ldots$ \\
\hline$k T_{\mathrm{TB} / \mathrm{MEK} / \mathrm{COM}}(\mathrm{keV})$ & $\ldots$ & $0.33 \pm 0.03(\mathrm{~TB})$ & $0.9_{-0.3}^{+4}(\mathrm{COM})$ & $\ldots$ & 0.37 (MEK) & $\ldots$ & $\ldots$ & $\ldots$ \\
\hline$k T_{\mathrm{BB}}$ plus $k T_{\mathrm{TB}}(\mathrm{keV})$ & $\ldots$ & $\ldots$ & $0.03-0.17,0.5-0.8$ & $\ldots$ & $\ldots$ & $0.30 \pm 0.01$ & $0.11 \pm 0.1$ & $(0.4-0.7) \pm 0.1$ \\
\hline Low-E line energies $(\mathrm{keV}) \ldots \ldots \ldots \ldots$ & 0.95 & $\ldots$ & $0.9,1.9$ & $\ldots$ & $\ldots$ & 1.05 & $\ldots$ & $\ldots$ \\
\hline
\end{tabular}

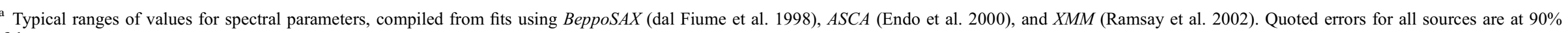
confidence.

b ROSAT/Ginga (Woo et al. 1995) and ASCA (Paul et al. 2002).

c ROSAT/Ginga (Woo et al. 1996) and BeppoSAX (La Barbera et al. 2001; Naik \& Paul 2004).

$A S C A$ (Yokogawa et al. 2000).

ROSAT/ASCA (Kohno et al. 2000).

BeppoSAX (Orlandini et al. 1998b)
BeppoSAX (Burderi et al. 2000).

hOSAT (Haberl et al. 1994), BeppoSAX (Orlandini et al. 1998a), and RXTE (Kreykenbohm et al. 2002).

The two values for $N_{\mathrm{H}}$ indicate interstellar absorption and partially covering absorption instrinsic to the source.

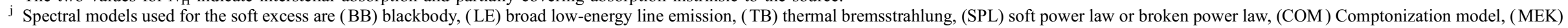
MEKAL thin thermal model, and (lines) resolved individual emission lines. Commas indicate separate fits; plus signs indicate fits with two components. 
system is at high inclination, and the A7 companion star, with a mass of $\sim 2 M_{\odot}$, eclipses the pulsar every orbital period (Middleditch \& Nelson 1976). In addition, there is a 35 day cycle in the X-ray intensity, consisting of "main-on" and "shorton" states separated by two "low" states in which the X-ray flux drops by several orders of magnitude (Jones \& Forman 1976). This modulation is believed to be due to a tilted precessing accretion disk that periodically blocks the central X-ray source (see Scott \& Leahy 1999 for a review). Given a distance of $5 \mathrm{kpc}$, Her X-1 has $L_{\mathrm{X}} \sim 2 \times 10^{37}$ ergs s$^{-1}$.

The spectrum of Her X-1 has been extensively studied and is generally fitted with a cutoff power law with $\Gamma \sim 1$, a blackbody with $k T_{\mathrm{BB}} \sim 0.1 \mathrm{keV}$, and often broad-line emission at $\sim 1 \mathrm{keV}$. There is also iron emission at $\sim 6.5 \mathrm{keV}$ and a cyclotron feature at $\sim 40 \mathrm{keV}$ (dal Fiume et al. 1998). The energy of the (fundamental) cyclotron line is related to the $B$ field by

$$
E_{\text {cyc }}=11.6\left(B / 10^{12} \mathrm{G}\right)(1+z)^{-1} \mathrm{keV},
$$

where $z$ is the gravitational redshift (i.e., Coburn et al. 2002). For surface emission from a neutron star with $R_{\mathrm{NS}}=10 \mathrm{~km}$ and $M=1.4 M_{\odot}$, we have $z \sim 0.3$ (Coburn et al. 2002). Usually neither $E_{\mathrm{cyc}}$ nor $z$ is well constrained, so for simplicity we take $B \simeq 10^{12}\left(E_{\text {cyc }} / 10 \mathrm{keV}\right) \mathrm{G}$ in this paper. Thus, Her X-1 has $B \sim 4 \times 10^{12} \mathrm{G}$.

The pulsations of the soft component are generally smoother than and out of phase with the hard component (McCray et al. 1982; Endo et al. 2000). This phase offset varies over the superorbital period (Ramsay et al. 2002), supporting the idea that the soft excess is emitted by the precessing accretion disk. We discuss this process in detail in $\S 7$.

\subsection{The Luminous Magellanic Cloud XBPS}

SMC X-1 is a luminous, eclipsing, high-mass XBP system. It was first identified as a point source in Uhuru observations by Leong et al. (1971). Eclipses were discovered by Schreier et al. (1972a) and X-ray pulsations by Lucke et al. (1976). The optical counterpart is Sk 160, a B0 I supergiant (Webster et al. 1972; Liller 1973). The orbit is close to circular, with orbital period $\sim 3.9$ days and pulse period $0.7 \mathrm{~s}$. The source has a high $L_{\mathrm{X}} \sim 2 \times 10^{38} \mathrm{ergs}^{-1}$. As in Her X-1 there are large, quasiperiodic variations of an $\sim 60$ day period between high and low states of the X-ray flux, attributed to blocking by a precessing disk (Gruber \& Rothschild 1984). These variations are stable, and the period of the cycles oscillates from $\sim 50$ to $\sim 60$ days (Wojdowski et al. 1998). This oscillation is itself periodic on the order of years (Clarkson et al. 2003). The spectrum of SMC X-1 is usually fitted as a cutoff power law with $\Gamma \sim 0.9$, an iron line at $\sim 6.4 \mathrm{keV}$, and a soft component modeled as a broken power law, thermal bremsstrahlung, or a blackbody with $k T_{\mathrm{BB}} \sim 0.16 \mathrm{keV}$. Paul et al. (2002) fitted four different models to the soft component and found that all gave satisfactory fits. Similarly to Her X-1, they found that the soft component pulses roughly sinusoidally, out of phase with the sharper peaked power law.

LMC X-4 is a high-mass XBP very similar to SMC X-1 and was also discovered by Uhuru observations (Giacconi et al. 1972). Its companion is an O III-O IV star (Sanduleak \& Philip 1977). It is eclipsing ( $\mathrm{Li}$ et al. 1978; White 1978) and has a pulse period of $13.5 \mathrm{~s}$ (Kelley et al. 1983) and an orbital period of 1.4 days. LMC X-4 also has a stable superorbital period of $\sim 30$ days, again attributed to a precessing disk (Lang et al. 1981). About once per day the source shows flares lasting 20-45 minutes, during which the X-ray flux increases by up to 20 times (e.g., Levine et al. 1991). Like SMC X-1, the high-state spectrum is fitted by a cutoff power law $(\Gamma \sim 0.6)$, with a soft component modeled in a variety of ways suggesting different emission mechanisms. In the low state, the power law hardens to $\Gamma \sim 0.1$ (Naik \& Paul 2002). There are iron emission at $\sim 6.5 \mathrm{keV}$ and a cyclotron feature at $\sim 100 \mathrm{keV}$ (La Barbera et al. 2001), giving $B \sim 10^{13}$ G. Similar to SMC X-1, the soft component pulses more smoothly than the hard component and is perhaps out of phase with it (Woo et al. 1996; Paul et al. 2002). For both SMC X-1 and LMC X-4, Paul et al. (2002) argue that the soft component is likely not thermal in origin and is best described by power-law emission from the upper accretion column.

XTE J0111.2-7317 is a bright transient XBP in the SMC with a probable Be star companion. It was discovered in 1998 November, simultaneously by RXTE (Chakrabarty et al. 1998) and BATSE (Wilson \& Finger 1998), and has a pulse period of $30.95 \mathrm{~s}$. The source showed two outbursts of $\sim 20$ and 40 days in duration, and the pulsar was spinning up with a very short timescale of $P / \dot{P} \sim 20$ yr (Yokogawa et al. 2000). The likely Be companion may be embedded in an $\mathrm{H}$ II region (Coe et al. 2000, 2003). Given a distance of $65 \mathrm{kpc}$ to the SMC, the source had a $0.7-10 \mathrm{keV}$ luminosity of $\sim 2 \times 10^{38} \mathrm{ergs} \mathrm{s}^{-1}$. The $A S C A$ spectrum is an inverse, broken power law with iron emission at $6.4 \mathrm{keV}$, along with a soft component that was modeled as a broken power law, thermal bremsstrahlung, or blackbody (Yokogawa et al. 2000). Variations in the soft X-ray band indicate that the soft component is pulsing.

RX J0059.2-7138 is another bright transient Be/XBP in the SMC. It was discovered as a serendipitous source in a short pointing of ROSAT (Hughes 1994). The ROSAT plus ASCA spectrum has luminosity $\sim 3 \times 10^{38} \mathrm{ergs} \mathrm{s}^{-1}$, and the hard component is fitted by a cutoff power law with $\Gamma \sim 0.4$ (Kohno et al. 2000). The soft component, for which $L_{\text {soft }} / L_{X} \sim 35 \%$, was fitted using either a soft broken power law in addition to the hard power law or a thin thermal emission model (MEKAL; Mewe et al. 1986) with $k T_{\mathrm{MEK}}=0.37 \mathrm{keV}$. Fitting this model required a very low value for overall metal abundance in the emitting gas $\left(Z=0.015 Z_{\odot}\right)$ but a highly enhanced oxygen abundance in the absorbing column $\left(Z_{\mathrm{O}} \sim 7 Z_{\odot}\right)$. Unlike the four sources discussed above, its soft component does not pulsate.

\subsection{Other Pulsars with a Soft Excess}

We know of three other well-studied XBPs that show a soft excess. 4U 1626-67, like Her X-1, is one of the few XBPs with a low-mass companion. X-ray pulsations with period $7.7 \mathrm{~s}$ were first detected by $S A S 3$ (Rappaport et al. 1977), and a faint blue optical counterpart was identified by McClintock et al. (1977). There is an unusually short orbital period of 41.4 minutes (Middleditch et al. 1981), which rules out a hydrogen-burning main-sequence star for the companion. In 1990 June the steady spin-up of the source suddenly changed to a spin-down state that continues to the present. The spectrum has repeatedly been fitted with a power law $(\Gamma \sim 0.7)$ plus a blackbody $\left(k T_{\mathrm{BB}} \sim 0.3 \mathrm{keV}\right)$ and emission-line features at $\sim 1 \mathrm{keV}$ (Angelini et al. 1995; Owens et al. 1997; Orlandini et al. 1998b; Schulz et al. 2001). The pulse fraction is much lower for soft than hard X-rays (Owens et al. 1997), suggesting that the soft component is not pulsing. Orlandini et al. (1998b) discovered a cyclotron absorption feature at $\sim 35 \mathrm{keV}$, implying that $B \sim 3.5 \times 10^{12} \mathrm{G}$. They found the broadband $(0.1-$ $200 \mathrm{keV}) \mathrm{X}$-ray luminosity to be $\left(7.7 \times 10^{34} \mathrm{ergs} \mathrm{s}^{-1}\right) D_{\mathrm{kpc}}^{2}$. The distance is not well constrained, so it is difficult to make 
reliable conclusions about the physical processes in this source.

There is also evidence for a soft excess in Cen X-3. It is a high-mass XPB, with pulse period of $4.8 \mathrm{~s}$ and orbital period of 2.1 days (Schreier et al. 1972b), and the O-type companion is estimated to be at a distance of $\sim 8 \mathrm{kpc}$ (Krzeminski 1974). The hard continuum has a typical cutoff power-law shape, with iron line emission at $\sim 6.5 \mathrm{keV}$ (Nagase et al. 1992; Burderi et al. 2000) and a cyclotron line feature at $\sim 30 \mathrm{keV}$ (Santangelo et al. 1998; Burderi et al. 2000; Coburn et al. 2002), giving $B \sim 3 \times 10^{12} \mathrm{G}$. Using BeppoSAX data, Burderi et al. (2000) found a bright pulsating soft excess with a blackbody shape $\left(k T_{\mathrm{BB}} \sim 0.1 \mathrm{keV}\right)$. In this observation Cen $\mathrm{X}-3$ had a large $L_{\mathrm{X}} \sim 10^{38} \mathrm{ergs} \mathrm{s}^{-1}$.

Vela X-1 also shows a soft excess. It has a long pulse period of $283 \mathrm{~s}$ (McClintock et al. 1976) and an orbital period of 8.96 days (Forman et al. 1973). The power-law spectrum has $\Gamma \sim 1-1.7$ (Sako et al. 1999; Choi et al. 1996), and the distance is $\sim 1.9 \mathrm{kpc}$ (Sadakane et al. 1985), indicating $L_{\mathrm{X}} \sim$ $10^{36} \mathrm{ergs} \mathrm{s}^{-1}$. There are cyclotron features at $\sim 24$ and $55 \mathrm{keV}$ (Kreykenbohm et al. 2002; Kretschmar et al. 1996), indicating $B \sim 2.4 \times 10^{12} \mathrm{G}$. The ROSAT spectrum showed a soft, nonpulsing thermal bremsstrahlung component with $k T_{\text {tb }} \sim$ $0.5 \mathrm{keV}$, attributed to shock-heated optically thin emission by the strong stellar wind (Haberl 1994). This was similar to the high-mass X-ray binary 4U 1700-37, which had a remarkably similar ROSAT soft excess spectrum (Haberl et al. 1994). However, high-resolution grating spectroscopy of these sources with Chandra showed that in eclipse there were emission lines that likely came from material that was not collisionally excited but was photoionized by the X-rays from the neutron star (Schulz et al. 2002; Boroson et al. 2003). We discuss collisionally heated and photoionized gas emission in $\S \S 5$ and 6 , respectively.

There are two additional transient Be/XBPs in the LMC for which a spectrally fitted soft excess has been seen. EXO 053109-6609.2 was the brightest source in a survey of the LMC by Haberl et al. (2003), who estimated $L_{X}=4.6 \times$ $10^{37} \mathrm{ergs} \mathrm{s}^{-1}$. They fitted the soft component as thin thermal (MEKAL) emission with reduced abundances $\left(0.29 Z_{\odot}\right)$ of elements heavier than oxygen. A0538-66 was seen in two outbursts with ROSAT (Mavromatakis \& Haberl 1993), when the source had $L_{\mathrm{X}} \sim 2-4 \times 10^{37} \mathrm{ergs} \mathrm{s}^{-1}$. They fitted the spectra with a power law, $\Gamma \sim 0.8$, a soft blackbody, $k T_{\mathrm{BB}} \sim 0.2 \mathrm{keV}$, and a thermal bremsstrahlung, $k T_{\mathrm{tb}} \sim 0.25$, although the spectra have a relatively low number of counts, so there may be considerable uncertainty in these parameters. The authors speculate that the blackbody component may be emitted from near the Alfvén radius of the neutron star, while the bremsstrahlung component may be powered by collisional heating of the stellar wind. Although we mention EXO 053109-6609.2 and A0538-66 here, they have little spectral information in soft $\mathrm{X}$-rays, so we do not discuss them in detail.

\section{UBIQUITY OF THE SOFT EXCESS}

In this section we address whether the soft excess is a universal feature of XBPs. All the brightest sources with low absorption show this feature, so it is natural to suspect that it may be present also in other XBPs but not be detected because of either low flux or high absorbing column. In Table 2 we show spectrally determined $N_{\mathrm{H}}$ values and the unabsorbed fluxes for a number of known XBPs. The sample is taken from Table 1 in Bildsten et al. (1997), plus EXO 053109-6609.2. We have included only sources for which there are observa- tions with sensitivity at the energies of the soft excess less than $2 \mathrm{keV}$. The fluxes are defined in different bands in the range $0.1-20 \mathrm{keV}$, so there is some error (factors of a few or so) in the relative fluxes from these sources, although this does not affect the overall pattern observed.

The sources in the sample are plotted as a function of flux and $N_{\mathrm{H}}$ in Figure 1. The XBPs with a known soft excess are shown as stars; those without, as squares. It is clear that the brighter systems with less absorption are far more likely to show a soft excess and that no sources with high absorption $\left(N_{\mathrm{H}}>3 \times 10^{22} \mathrm{~cm}^{-2}\right)$ show this feature. There is an apparent boundary between the sources that show a soft excess and those that do not, suggesting that the observability of this feature depends on flux and $N_{\mathrm{H}}$.

Marked with an asterisk is the Be/XBP X Persei, which has a markedly different soft excess from the other sources. Coburn et al. (2001) modeled this component as a blackbody with $k T_{\mathrm{BB}} \sim 1.4 \mathrm{keV}$, which is an order of magnitude higher than the typical temperature. X Per is very nearby $(D \sim 0.7 \mathrm{kpc})$ and underluminous $\left(L_{\mathrm{X}} \sim 10^{34} \mathrm{ergs} \mathrm{s}^{-1}\right.$; di Salvo et al. 1998), and the authors attributed the soft component to blackbody emission from the accreting polar cap.

We modeled the detectability of the soft excess at various fluxes and $N_{\mathrm{H}}$ by performing fits to simulated data. The response of the ROSAT PSPC detector (Pfeffermann \& Briel 1986 ) is used, because many XBPs have been observed with this detector and because it is sensitive in the soft X-ray band. Using XSPEC (Arnaud 1996), we created simulated PSPC spectra of a source with a spectrum like that of SMC X-1 ( $\Gamma=0.9, k T_{\mathrm{BB}}=0.15 \mathrm{keV}$, and $L_{\mathrm{soft}} / L_{\mathrm{X}}=0.04$ ) and varied $N_{\mathrm{H}}$ and the overall unabsorbed flux in the $0.2-3 \mathrm{keV}$ band. The simulated exposure time was $25 \mathrm{ks}$, and counting statistics were included. We also included a typical background spectrum, taken from the PSPC observation of SMC X-1 from 1991 October. This background has a surface brightness in the PSPC band $(0.1-2.4 \mathrm{keV})$ of $2.1 \times 10^{-2}$ counts $\mathrm{s}^{-1} \operatorname{arcmin}^{-2}$, which is similar to those found for Cen X-3, LMC X-4, and Her X-1. More details on PSPC observations of these sources are given in $\S 4.1$.

Next, we determined whether each spectrum, after background subtraction, could be fitted with an absorbed power law without a soft excess component. For the fits we took initial values of $N_{\mathrm{H}}$ and $\Gamma$ and overall normalization from the simulated data and allowed all these parameters to vary. We recorded $\chi_{\nu}^{2}$ for the best fit to each simulation. For low $\chi_{\nu}^{2}$, we conclude that a power-law spectrum satisfactorily fits the simulated data, and so the soft excess is not detectable. For high $\chi_{\nu}^{2}$ the soft excess is detectable.

The fit results are plotted in Figure 2, along with the XBPs from Figure 1. Contours of $\chi_{\nu}^{2}=1.2,1.5$, and 2 are shown. For low flux and high $N_{\mathrm{H}}$ (bottom right), $\chi_{\nu}^{2}$ varies somewhat randomly between 0.8 and 1.2 ; for clarity, these contours are not shown. We find that the soft excess is detectable in sources with high flux and low $N_{\mathrm{H}}$ and undetectable for low flux and high $N_{\mathrm{H}}$. The steep contours of $\chi_{\nu}^{2}$, which indicate the boundary of detectability, are close to and similar in shape to the actual boundary seen in observations. This suggests that many sources with low flux or high $N_{\mathrm{H}}$ do in fact have a soft component but that this feature has not been observed because of selection effects. We conclude that the soft excess is probably a very common intrinsic feature of XBPs.

As discussed in $\S 2$, observations of the soft excess in XBPs have been modeled in a variety of ways, many of which require different emission processes. Given the similarities of many of 
TABLE 2

Unabsorbed Flux and $N_{\mathrm{H}}$ Values for a Sample of XBPs

\begin{tabular}{|c|c|c|c|c|c|}
\hline Source & $\begin{array}{c}\text { Flux } \\
\left(\operatorname{ergs~} \mathrm{cm}^{-2} \mathrm{~s}^{-1}\right)\end{array}$ & $\begin{array}{c}\text { Energy Range } \mathrm{e}^{\mathrm{a}} \\
(\mathrm{keV})\end{array}$ & $\begin{array}{c}N_{\mathrm{H}} \\
\left(10^{22} \mathrm{~cm}^{-2}\right)\end{array}$ & Mission & Reference \\
\hline \multicolumn{6}{|c|}{ Soft Excess Observed } \\
\hline 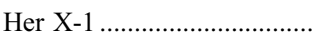 & $3.7 \times 10^{-8}$ & $0.3-10$ & 0.005 & $X M M$ & 1 \\
\hline 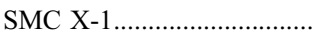 & $4.7 \times 10^{-10}$ & $0.7-10$ & 0.5 & $A S C A$ & 2 \\
\hline LMC X-4 …...................... & $4.0 \times 10^{-10}$ & $0.7-10$ & 0.057 & $A S C A$ & 2 \\
\hline XTE $0111.2-7317 \ldots \ldots \ldots \ldots . . .$. & $3.6 \times 10^{-10}$ & $0.7-10$ & 0.18 & $A S C A$ & 3 \\
\hline RX J0059.2-7138 ................ & $6.9 \times 10^{-11}$ & $0.2-2$ & 0.088 & ROSAT & 4 \\
\hline 4U 1626-67 ........................... & $6.4 \times 10^{-10}$ & $0.5-10$ & 0.5 & Chandra & 5 \\
\hline 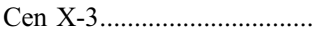 & $5.7 \times 10^{-9}$ & $2.0-10$ & 1.95 & BeppoSAX & 6 \\
\hline Vela X-1 …............................ & $5.1 \times 10^{-9}$ & $2.0-10$ & 0.86 & BeppoSAX & 7 \\
\hline EXO 053109-6609.2 .......... & $1.5 \times 10^{-10}$ & $0.2-10$ & 0.69 & $X M M$ & 8 \\
\hline A0538-66 & $1.3 \times 10^{-10}$ & $0.1-2.4$ & 0.08 & ROSAT & 9 \\
\hline \multicolumn{6}{|c|}{ Soft Excess Not Observed } \\
\hline GRO J1744-28 …….......... & $2.3 \times 10^{-8}$ & $2.0-10$ & 5 & $A S C A$ & 10 \\
\hline GX $1+4$ & $8.4 \times 10^{-10}$ & $2.0-20$ & 20 & $A S C A$ & 11 \\
\hline 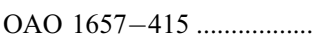 & $4.7 \times 10^{-10}$ & $2.0-10$ & 12 & BepposAX & 12 \\
\hline 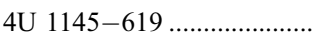 & $5.0 \times 10^{-11}$ & $0.2-2$ & 0.3 & EXOSAT & 13 \\
\hline 4U 1907+09 & $4.0 \times 10^{-10}$ & $2.0-10$ & 1.9 & $A S C A$ & 14 \\
\hline 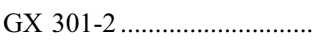 & $4.6 \times 10^{-8}$ & Bolometric & 20 & $A S C A$ & 15 \\
\hline 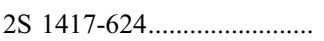 & $1.7 \times 10^{-10}$ & $2.0-10$ & 1.7 & Einstein & 16 \\
\hline GRO J1008-57 .................... & $2.3 \times 10^{-11}$ & $0.1-2.4$ & 1.3 & ROSAT & 17 \\
\hline 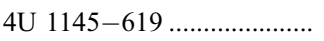 & $5.0 \times 10^{-11}$ & $0.2-2$ & 0.3 & EXOSAT & 14 \\
\hline X Per ................................................ & $2.0 \times 10^{-10}$ & $0.1-10$ & 0.13 & BeppoSAX & 18 \\
\hline
\end{tabular}

${ }^{a}$ Energy range over which the flux is defined.

References.-(1) Ramsay et al. 2002; (2) Paul et al. 2002; (3) Yokogawa et al. 2000; (4) Hughes 1994; (5) Schulz et al. 2001; (6) Burderi et al. 2000; (7) Orlandini et al. 1998a; (8) Haberl et al. 2003; (9) Mavromatakis \& Haberl 1993; (10) Nishiuchi et al. 1999; (11) Kotani et al. 1999; (12) Orlandini et al. 1999; (13) Mereghetti et al. 1987; (14) Roberts et al. 2001; (15) Endo et al. 2002; (16) Grindlay et al. 1984; (17) Petre \& Gehrels 1994; (18) di Salvo et al. 1998.

the observed soft components and the apparent ubiquity of this feature, it is likely that it has a common origin in many sources. Possible mechanisms can be separated into four main types: (1) emission from the accretion column, (2) thermal emission from collisionally energized diffuse gas around the neutron star, (3) reprocessing of the hard (power law) X-rays by diffuse material around the neutron star, and (4) reprocessing by optically thick, dense material around the neutron star.

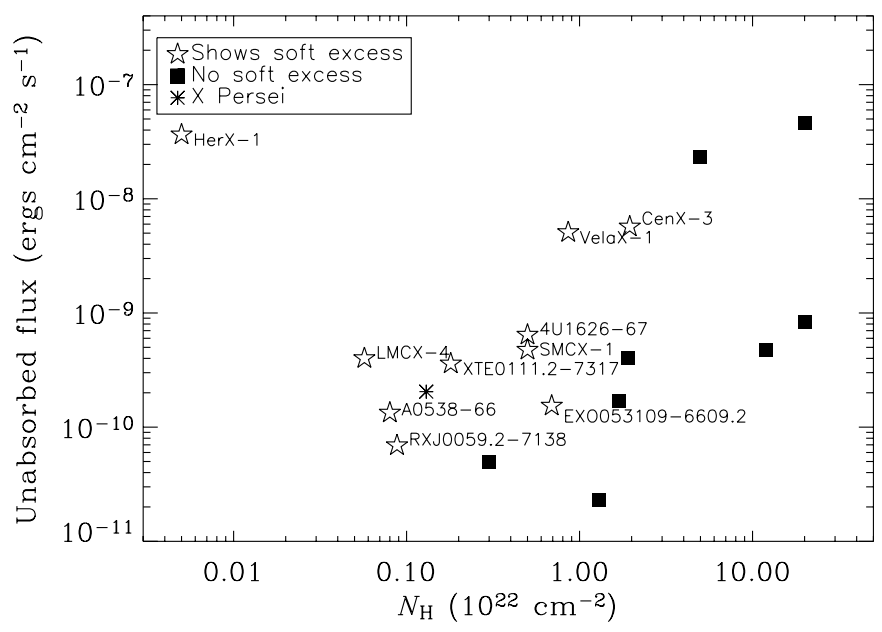

Fig. 1.-Observed $N_{\mathrm{H}}$ and unabsorbed fluxes for the XBPs in Table 1 of Bildsten et al. (1997) plus EXO 053109-6609.2. Sources with a known soft excess are shown as stars, while those without are shown as squares. The lowluminosity source X Per is shown as an asterisk (see text for details).
In the following sections, we consider these four scenarios and examine them in light of observational data and the physics of the relevant emission. Simple schematics of the various models are shown in Figure 3. For a model to be viable it must satisfy two constraints: (1) the energy balance of the system must be able to produce the observed soft luminosity and

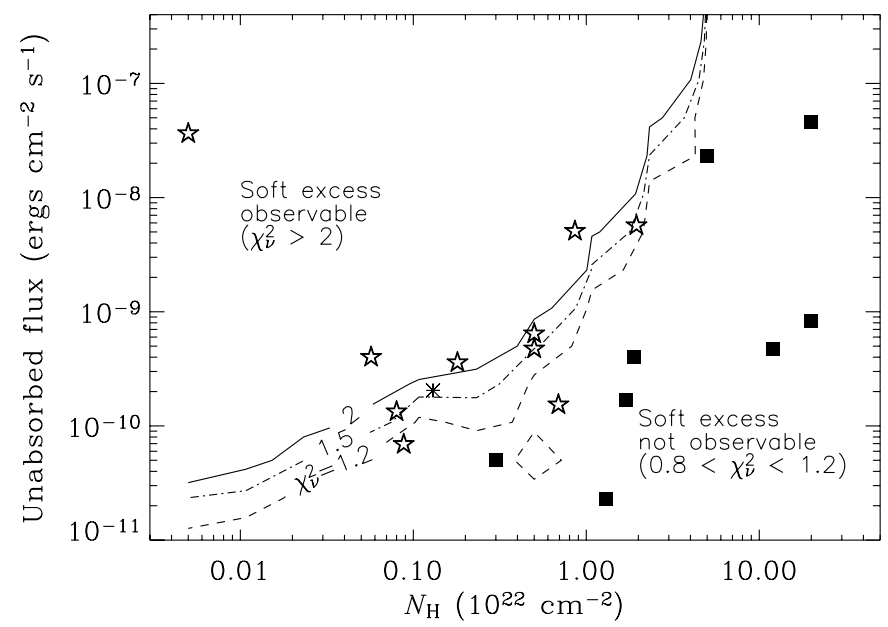

FIG. 2.-Observability of the soft excess as a function of $N_{\mathrm{H}}$ and unabsorbed fluxes, from fits to simulated ROSAT data. Contours of $\chi_{\nu}^{2}$ for fits to a simple absorbed power law are shown. The soft excess is observable for high $\chi_{\nu}^{2}$ and not observable for low $\chi_{\nu}^{2}$. The XBPs from Fig. 1 are also shown for comparison; note that the $\chi_{\nu}^{2}$ contours are similar in shape to the boundary between observed XBPs with and without a soft excess. 
Process 1: Emission from the accretion column

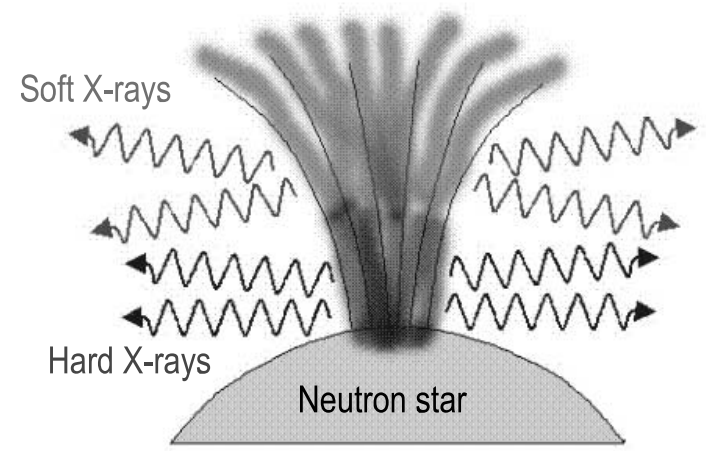

Process 4: Reprocessing by optically thick material

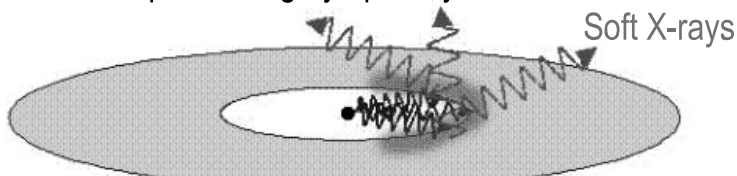

Accretion disk
Process 2: Collisionally energized emission

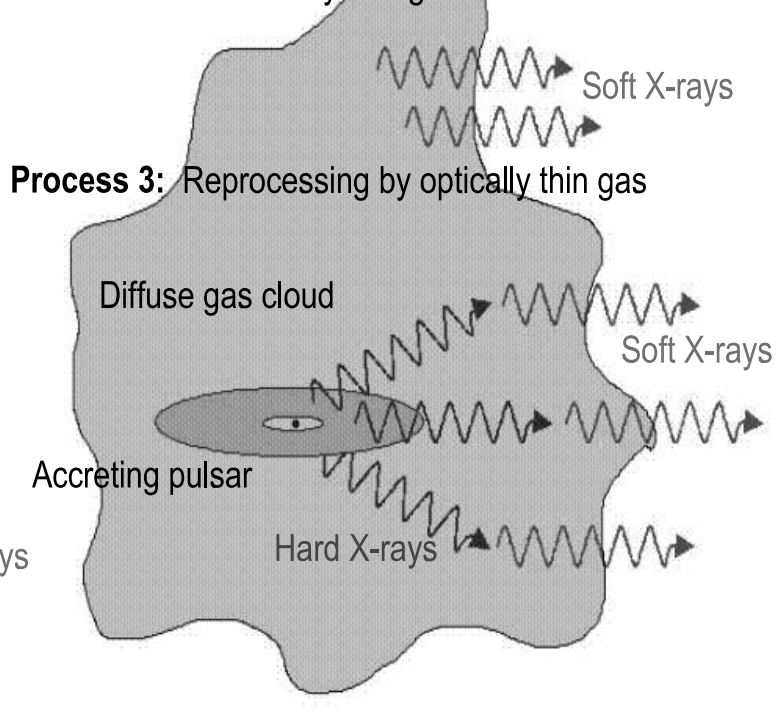

FIG. 3.-Four soft excess emission processes considered in this paper.

(2) the spectrum, pulsation properties, and other observed features must be consistent with model predictions.

\section{EMISSION FROM THE ACCRETION COLUMN}

It is natural to suspect that the soft component originates in the same region as the hard component, that is, in the column of accreting gas. Low-luminosity soft emission from the polar cap may exist in X Per (di Salvo et al. 1998), and the more luminous soft components in SMC X-1, LMC X-4, and XTE J0111.2-7317 have been modeled by nonthermal broken power-law emission from the accretion column (Yokogawa et al. 2000; Paul et al. 2002). This model predicts soft pulses, because emission is confined to a small region that rotates with the neutron star.

\subsection{Brightness Temperatures of Accretion Columns}

In XBPs the accreting material flows along magnetic field lines from the magnetosphere, at radius $R_{m}$, to the surface of the neutron star at $R_{\mathrm{NS}}$. To estimate the surface area of the accretion column, we follow $\S 6.3$ of Frank et al. (2002). We assume that the magnetic field is a dipole that is tilted by an angle $\alpha$ with respect to the accretion disk and that only field lines that pass through the disk outside $R_{m}$ accrete. It is straightforward to show that all the accreting field lines intersect the neutron star inside an angle $\beta$ from the pole, where

$$
\sin ^{2} \beta=\left(R_{\mathrm{NS}} / R_{m}\right) \sin ^{2} \alpha .
$$

Thus, the radius of the accreting polar cap is

$$
R_{\mathrm{col}} \simeq R_{\mathrm{NS}} \sin \beta \sim R_{\mathrm{NS}}\left(R_{\mathrm{NS}} / R_{m}\right)^{1 / 2}
$$

assuming $\sin \alpha \sim 1$. For the XBPs we consider, $R_{m} \sim 10^{8} \mathrm{~cm}$, so for $R_{\mathrm{NS}} \sim 10^{6} \mathrm{~cm}, R_{\mathrm{col}} \sim 0.1 R_{\mathrm{NS}}$. If we picture the accretion column as a cylinder with height $\lesssim R_{\mathrm{NS}}$, the surface area of the column is

$$
A_{\mathrm{col}} \lesssim 2 \pi R_{\mathrm{col}} R_{\mathrm{NS}}=f_{\mathrm{col}} 4 \pi R_{\mathrm{NS}}^{2} .
$$

Here $f_{\text {col }}$ is simply the surface area of the accretion column as a fraction of the neutron star's surface area, with $f_{\text {col }} \lesssim 0.1$.

For high $L_{\text {soft }}$, this small area implies a very high temperature for the emitting gas. The minimum temperature for material radiating a spectral luminosity $L_{\nu}$ in a given band is the brightness temperature $T_{b}$, which is the temperature of a blackbody that would produce the same $L_{\nu}$. For an emitting area $A$,

$$
L_{\nu}=A \pi B_{\nu}\left(T_{b}\right)
$$

The value of $T_{b}$ for different energy ranges sets a lower limit on the temperature of the gas emitting at those energies.

We used data from the ROSAT PSPC observations for Her $\mathrm{X}-1$, SMC X-1, LMC X-4, and Cen X-3, as well as RX J0059.2-7138 and Vela X-1, which we include for comparison, even though their lack of soft pulsations would appear to rule out accretion column emission. Data on these observations are given in Table 3. The PSPC is a proportional counter detector with response at low energies, from 0.1 to $2.4 \mathrm{keV}$, and with spectral resolution $\Delta E / E=0.43(E / 0.93 \mathrm{keV})^{-1 / 2}$ (Pfeffermann \& Briel 1986). We obtained the data from the NASA HEASARC archive and used the standard eventscreening criteria. We extracted the source and background spectra from circular and annular regions centered on the

\begin{tabular}{|c|c|c|c|}
\hline Source & Date & $\begin{array}{c}\text { Duration } \\
(\mathrm{ks})\end{array}$ & $\begin{array}{c}N_{\mathrm{H}} \\
\left(10^{22} \mathrm{~cm}^{-2}\right)\end{array}$ \\
\hline 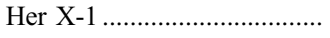 & 1993 Aug & 5 & 0.003 \\
\hline 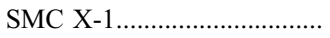 & 1991 Oct & 17 & 0.36 \\
\hline 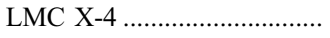 & 1991 Oct-Nov & 33 & 0.05 \\
\hline RX J0059.2-7138 .............. & 1993 May & 5 & 0.04 \\
\hline 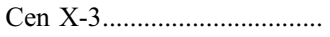 & 1992 Jan & 9 & 2 \\
\hline 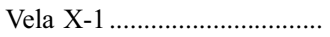 & 1992 Nov & 17 & 0.5 \\
\hline
\end{tabular}
source. In the case of RX J0059.2-7138, a nearby supersoft

TABLE 3

ROSAT PSPC Data Used for $T_{b}$ Calculations 
source (1E 0056.8-7154) was excluded from the selection regions, as described in Kohno et al. (2000).

We assume that all the observed flux in the spectrum comes from a column of area $A_{\mathrm{col}}$ and determine $T_{b}$ as a function of energy. We use energy bins corresponding to the PSPC resolution and fit each bin with a power law of constant energy (photon $\Gamma=1$ ), modified by typical values of $N_{\mathrm{H}}$ (shown in Table 3). Each XSPEC fit gives the normalization of the powerlaw $I_{p l}$ (in photons $\mathrm{cm}^{-2} \mathrm{~s}^{-1} \mathrm{keV}^{-1}$ at $1 \mathrm{keV}$ ) and its error. The energy flux in each bin (from $E_{1}$ to $E_{2}$ ) is then

$$
F_{\mathrm{obs}}=\int_{E_{1}}^{E_{2}} E\left(I_{\mathrm{pl}} E^{-1}\right) d E=I_{\mathrm{pl}}\left(E_{2}-E_{1}\right) .
$$

Given the distances listed in Table 1, we calculate the luminosities and then $T_{b}$ in each bin. To obtain a lower limit on $T_{b}$, we use the $3 \sigma$ lower limit on $I_{\mathrm{pl}}$.

The isotropic luminosity observed in a given bin is

$$
L_{\mathrm{obs}}=4 \pi D^{2} F_{\mathrm{obs}}
$$

and the luminosity emitted from the accretion column in that bin is

$$
L_{\mathrm{em}}=A_{\mathrm{col}} \pi \int_{E_{1}}^{E_{2}} B\left(T_{b}\right) d E
$$

Letting $L_{\mathrm{obs}}=L_{\mathrm{em}}$ and using $A_{\mathrm{col}}=f_{\mathrm{col}} 4 \pi R_{\mathrm{NS}}^{2}$, we have

$$
\int_{E_{1}}^{E_{2}} B\left(T_{b}\right) d E=\frac{D^{2}}{\pi R_{\mathrm{NS}}^{2} f_{\mathrm{col}}} F_{\mathrm{obs}} .
$$

We solve this numerically to find $T_{b}$. In the Rayleigh-Jeans limit $E \ll k T_{b}$, this gives, for $f_{\text {col }}=0.1, R_{\mathrm{NS}}=10 \mathrm{~km}, E_{1}$ and $E_{2}$ in $\mathrm{keV}$, and $L_{38}$, the luminosity in the bin in $10^{38} \mathrm{ergs} \mathrm{s}^{-1}$,

$$
T_{b}=\frac{L_{38}}{E_{2}^{3}-E_{1}^{3}} 1.75 \times 10^{10} \mathrm{~K} .
$$

For each source we plot the values of $T_{b}$, given $f_{\text {col }}=0.1$, in Figure 4. Vela X-1 and Cen X-3 are highly absorbed below $0.5 \mathrm{keV}$, so we do not calculate $T_{b}$ for those energies.

As expected, $T_{b}$ is higher for more luminous sources. For low energies, we find that for SMC X-1, LMC X-4, and RX J0059.2-7138, the observed flux gives $T_{b} \gtrsim 10^{11} \mathrm{~K}$, even for a relatively large accretion column area $\left(f_{\mathrm{col}}=0.1\right)$. At higher energies, we have $T_{b}>10^{8} \mathrm{~K}$ (and, remarkably, $T_{b}>10^{9} \mathrm{~K}$ for SMC X-1). These $T_{b}$ values represent the minimum possible temperatures of the gas emitting at these energies. Are these temperatures consistent with models of accretion columns? In general, such models have been separated into two types, depending on the shock mechanism that decelerates the gas as it approaches the neutron star's surface. The shock can be dominated by gas pressure (for low $L_{\mathrm{X}}$ systems) or by radiation pressure (for $L_{\mathrm{X}}>10^{36} \mathrm{ergs} \mathrm{s}^{-1}$ ). The latter models are applicable for the luminous XBPs. This type of accretion column consists of a mound of subsonic settling material below a radiation-dominated shock front (Becker 1998). Burnard et al. (1991) modeled the dynamics and emission properties of the accretion mounds and found that temperatures in the mound vary from $10^{8} \mathrm{~K}$ at the outer boundary to $2 \times 10^{8} \mathrm{~K}$ at large optical depths.

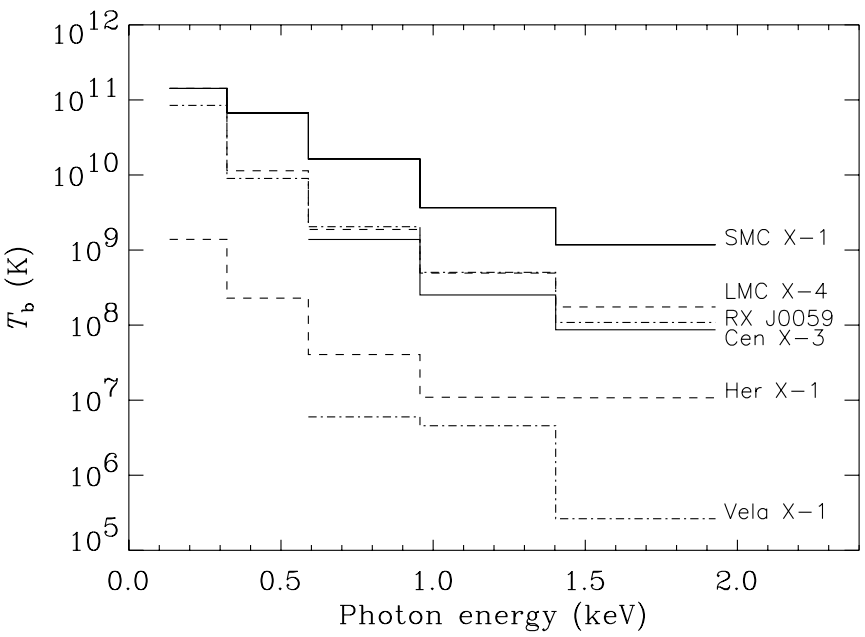

FIG. 4.-Brightness temperatures for the observed fluxes ( $3 \sigma$ lower limit) from ROSAT PSPC data of a sample of XBPs. We assume an emission region with area a fraction $f_{\mathrm{col}}=0.1$ of the surface area of the neutron star $\left(R_{\mathrm{NS}}=\right.$ $10 \mathrm{~km})$. For $E \ll T_{b}, T_{b} \propto f_{\text {col }}^{-1}$.

Burnard et al. (1991) note that the accretion mound emission is Compton-scattered by the overlying shock, which could increase $T_{b}$. In principle, if the gas free-falls all the way to the neutron star's surface and is stopped in a thin shock, then temperatures of $\sim 10^{12} \mathrm{~K}$ are possible (Shapiro \& Teukolsky 1983). However, we expect that collisions in the column would slow the gas, and it is expected that a radiative shock is not perfectly thin but has width approximately equal to the radius of the polar cap (Becker 1998). Therefore, we expect the shock temperature to be lower. It is difficult to see how Comptonization by a real shock could produce the very high soft fluxes observed.

This result raises the interesting, puzzling question of how, for SMC X-1, even the hard emission above $1.5 \mathrm{keV}$ could be produced by the accretion column. For a large column size $f_{\text {col }}=0.1$, the $T_{b} \sim 10^{9} \mathrm{~K}$ is hotter than models predict. This suggests that either the column is larger in extent (i.e., $f_{\text {col }}>$ $0.1)$ or it is more energetic $\left(T \gtrsim 10^{9} \mathrm{~K}\right)$ than predicted. This question is worth investigating in future work.

\subsection{Comparison with Cyclosynchrotron Spectra}

Even if accretion column temperatures are very high, it is difficult to produce the observed soft spectral shapes. For high $T_{b} \gtrsim 10^{7} \mathrm{~K}$, blackbody or thermal bremsstrahlung emission would produce the majority of the flux at energies $\sim k T>1$ $\mathrm{keV}$, which is inconsistent with observations. One possible way out is to note that, in the large magnetic field of the accretion column, the dominant emission process is likely to be cyclosynchrotron radiation. If this radiation is thermal, the peak of the emission can be at much lower energies than $k T$. The shape of this spectrum has been calculated in rigorous detail by Mahadevan et al. (1996), who show that it is a function of $\chi=E / E_{B}$, where

$$
E_{B}=\frac{h e B}{2 \pi m_{e} c}=11.6\left(B / 10^{12} \mathrm{G}\right) \mathrm{keV}
$$

is the (nonredshifted) cyclotron energy. Therefore, the energy range of cyclosynchrotron emission depends largely on $B$. We plot the calculated spectra from Mahadevan et al. (1996) in Figure 5. 


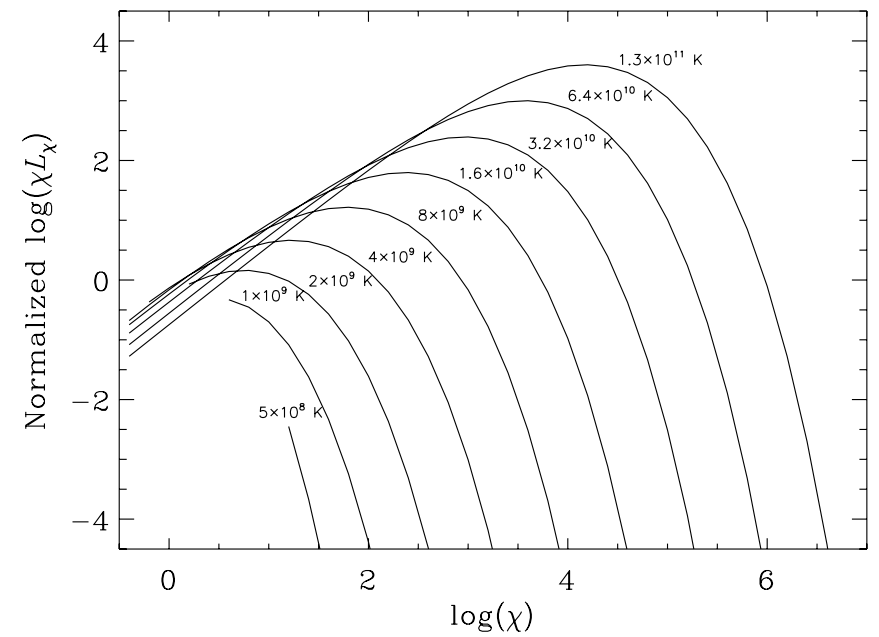

FIG. 5.-Spectra of cyclosynchrotron radiation for different temperatures, taken from Mahadevan et al. (1996). Spectra are plotted as a function of $\chi=E / E_{B}$.

At $T=3.2 \times 10^{10} \mathrm{~K}$, which is smaller than the minimum $T_{b}$ that we obtained for the luminous XBPs at low energies, the spectrum peaks at $\log \chi \sim 3$. A typical soft component in the luminous XBPs has the peak of $\nu F_{\nu}$ at $\sim 4 k T_{\mathrm{BB}} \sim 0.7 \mathrm{keV}$. For cyclosynchrotron emission to peak at this energy, we require that

$$
E_{B} \sim 0.7 \mathrm{keV} / \chi \sim 7 \times 10^{-4} \mathrm{keV}
$$

implying that $B \sim 6 \times 10^{7} \mathrm{G}$. For a dipole field with surface strength $4 \times 10^{12} \mathrm{G}$,

$$
B(r) \sim 4 \times 10^{12}\left(r / R_{\mathrm{NS}}\right)^{-3}
$$

implying that the emission must come from a radius $r \sim 40 R_{\mathrm{NS}}$. This is much larger than the expected size of the accretion column, and the gas cannot attain such a high temperature so far from the neutron star.

It is difficult to conceive of any other emission mechanism that could produce a high soft luminosity from a small area and yet have the spectrum cut off at the energies observed. We conclude that in the luminous XBPs (SMC X-1, LMC X-4, RX J0059.2-7138, XTE J0111.2-7317, and Cen X-3), the soft component must be emitted from a region larger than the surface of the neutron star.

\section{EMISSION BY A COLLISIONALLY ENERGIZED CLOUD}

A large diffuse cloud of gas around the neutron star would have the size needed to produce the high $L_{\text {soft }}$ and might be powered by collisional energy. Such emission may exist in Vela X-1 and 4U 1700-37 (Schulz et al. 2002; Boroson et al. 2003) and perhaps also in RX J0101.3-7211 and AX J0103-722, which were observed in the $X M M$ survey of the SMC by Sasaki et al. (2003). These sources have $L_{X} \sim 10^{35} \mathrm{ergs} \mathrm{s}^{-1}$ and were fitted with a power law plus an optically thin thermal emission model (MEKAL) with $k T_{\text {MEK }} \sim 0.2 \mathrm{keV}$.

Collisional heating is expected in XBP systems; a detailed hydrodynamic simulation of LMC X-4 by Boroson et al. (2001) found shocks and wake structures that could heat the gas and cause optically thin X-ray emission (Fransson \& Fabian 1980). Fitting a thin thermal emission model to data from SMC X-1 and LMC X-4, Paul et al. (2002) found that the observed high $L_{\text {soft }}$ requires $n^{2} V \sim 10^{61}$, which for typical gas density $n<10^{12} \mathrm{~cm}^{-3}$ implies a very large emission region of $R_{\text {cloud }}>10^{12} \mathrm{~cm}$. The same is true for XTE J0111.2-7317 and RX J0059.2-7138, which have similar soft spectra.

The large emission region immediately rules out this process for sources with a soft component with short pulsations, such as SMC X-1 and XTE J0111.2-7317. A pulse period of about a few seconds is much shorter than the light-crossing time of such a large cloud, and so the cloud's emission could not pulsate. For SMC X-1 and LMC X-4, the size of the emitting region is also constrained by the drop of the soft flux during eclipses (Vrtilek et al. 2001; Boroson et al. 2001). The eclipse light curves for SMC X-1 and LMC X-4 (e.g., Woo et al. 1995; La Barbera et al. 2001) show that the soft flux falls with a timescale $\lesssim 10 \%$ of the total eclipse duration. Thus, $R_{\text {cloud }}<$ $0.1 R_{*} \lesssim 10^{11} \mathrm{~cm}$. Such arguments rule out collisionally energized emission for SMC X-1, LMC X-4, and XTE J0111.27317 , but the process may still be important in RX J0059.27138, which shows neither eclipses nor pulsation at soft energies.

In XBPs the main input of kinetic energy is from a wind, simply $\dot{E}=\dot{M} v_{w}^{2} / 2$, where $v_{w}$ is the wind velocity, typically $\sim 2000 \mathrm{~km} \mathrm{~s}^{-1}$. Mass-loss rates from the star and from the irradiated accretion disk are typically $\dot{M} \lesssim 10^{-6} M_{\odot} \mathrm{yr}^{-1}$, giving $\dot{E} \lesssim 5 \times 10^{36} \mathrm{ergs} \mathrm{s}^{-1}$. Such emission can easily power the soft components of lower luminosity XBPs such as Vela X-1. However, it is possible for the more luminous soft excess of RX J0059.2-7138 only if there is a very vigorous wind for which all the kinetic energy is converted to soft X-rays. This is unlikely.

In addition, diffuse thermal emission in RX J0059.2-7138 should show line emission from metals. In their MEKAL fit to the $A S C A$ spectrum, Kohno et al. (2000) obtain an extremely low $Z=0.02$, which fits best because it greatly reduces the flux of most X-ray lines. While the metallicity of the SMC is expected to be low, this value is unrealistic. It is more likely that the nondetection of lines is due to optically thick (blackbody-like) emission, which we discuss in $\S 7$.

We conclude that collisionally energized diffuse emission can power the soft excess only in systems with $L_{\mathrm{X}} \lesssim 10^{36} \mathrm{ergs}$ $\mathrm{s}^{-1}$. This may explain part of the soft components in Vela X-1 and $4 \mathrm{U} 1700-37$, but in RX J0059.2-7138 the soft excess is too luminous for this process. With high spectral resolution observations, it is possible to easily identify this type of emission by the presence of X-ray emission lines. In addition, there should be other spectral signatures from diffuse gas around XBPs, because of photoionization of the gas by the central $\mathrm{X}$-ray source. We discuss this process in the next section.

\section{REPROCESSING BY A DIFFUSE CLOUD}

It is possible that the soft flux arises from reprocessing of the hard X-rays by a diffuse cloud of gas around the neutron star. Such a cloud must absorb and reradiate a fraction of the incident hard luminosity equal to $f_{\text {rep }}=L_{\text {soft }} / L_{\mathrm{X}} \sim 0.1$ and must produce an output spectrum like those observed in XBPs. Because of the high $L_{X}$ in these systems, the gas is highly photoionized by the X-ray flux. Emission from such a gas may contribute to the soft excess in Vela X-1 and 4U 1700-37 (Boroson et al. 2003; Schulz et al. 2002). The XSTAR code is designed to calculate the detailed structure of photoionized gas and has been used extensively to model the circumstellar environments in XBPs. For details on the code and its applications, see Kallman \& Bautista (2001). 
XSTAR works by determining the temperature, ionization, and excitation structure of a spherical gas cloud illuminated by a central point source of X-rays. It calculates the absorption by the gas due to photoionization and Compton heating and the emission from the gas through recombination, free-free emission, and atomic line emission. In the case of an optically thin low-density $\left(<10^{12} \mathrm{~cm}^{-3}\right)$ gas, the structure is a function simply of the ionization parameter $\xi=L / n R^{2}$, where $L$ is the luminosity of the central source, $n$ is the number density of the gas, and $R$ is the distance from the source (Tarter et al. 1969).

XSTAR has been used by Wojdowski et al. (2000) and Boroson et al. (2001) to compare the observed eclipse spectra from SMC X-1 and LMC X-4 with hydrodynamic simulations. Vrtilek et al. (2001) used XSTAR to analyze relatively low luminosity $\left(\sim 10^{35} \mathrm{ergs} \mathrm{s}^{-1}\right)$ photoionized line emission from SMC X-1 seen in Chandra ACIS spectra. In eclipse and in the low state (when the neutron star was blocked by the disk), they found lines that correspond to XSTAR simulations with $1<\log \xi<1.5$. Vrtilek et al. (2001) found that at a radius of $2 \times 10^{12} \mathrm{~cm}$ from the neutron star, these values for $\xi$ indicate a gas density of $\sim 10^{12} \mathrm{~cm}^{-3}$.

For most of the XBPs with $L_{\mathrm{X}} \gtrsim 10^{38} \mathrm{ergs} \mathrm{s}^{-1}$, we do not expect a diffuse cloud to be the source of the soft excess as discussed in $\S 5$. However, such a scenario is possible in RX J0059.2-7138, which does not show soft pulsations or eclipses, and so we test the possibility that optically thin reprocessing is the main source of the soft excess in this system. We have used the latest version of XSTAR, version $2.1 \mathrm{k}$, to simulate the photoionized gas in this source.

\subsection{XSTAR Input}

Because our models must reprocess a substantial fraction of the incident X-ray flux, we required that the intervening gas subtend a large solid angle around the central source. We thus modeled the reprocessing by spherical shells of gas centered on the neutron star. For a cloud of radial extent less than $10^{11} \mathrm{~cm}$, column densities higher than $10^{23} \mathrm{~cm}^{-2}$ imply densities higher than $10^{12} \mathrm{~cm}^{-3}$, for which we cannot parameterize our models simply in terms of $\xi$.

Instead, we constructed a series of models of gas in spherical shells with varying radius $R$ and column density $N$ (Fig. 6). Because the gas density in the system falls off with radius, the largest clouds of constant density should have thickness comparable to the scale length, or $\Delta R \sim R$. We therefore ran models with $\Delta R=R$ and $0.1 R$ to explore the effects of higher density parcels.

For the input spectrum we used an exponentially cutoff power law that matches the hard component of RX J0059.27138 as fitted by Kohno et al. (2000). The spectrum has luminosity $L_{\mathrm{X}}=2.6 \times 10^{38} \mathrm{ergs} \mathrm{s}^{-1}, \Gamma=0.43, E_{\text {cut }}=6.4 \mathrm{keV}$, and $E_{\text {fold }}=9.3 \mathrm{keV}$. For gas composition we used a standard SMC value of $Z=0.25$ (Welty 2001), as well as $Z=0.01$ and 1 .

There are large turbulent velocities in the gas around the neutron star, so we modified the standard XSTAR code to include these effects in the line opacity. For each model we set the turbulent velocities equal to the Keplerian velocity,

$$
v_{\text {turb }}=v_{\mathrm{K}}=1.9 \times 10^{4}\left(R / 10^{8} \mathrm{~cm}\right)^{-1 / 2} \mathrm{~km} \mathrm{~s}^{-1}
$$

for a neutron star mass of $1.4 M_{\odot}$. This decreases the line opacities by a factor of $v_{\text {thml }} /\left(v_{\text {turb }} \sqrt{A}\right)$, where $A$ is the atomic mass.

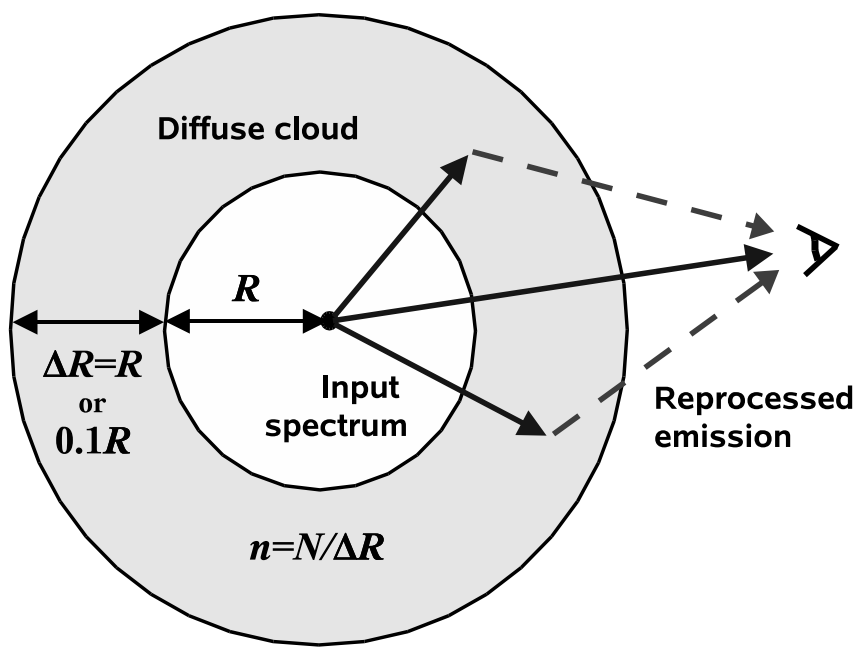

FIG. 6.-XSTAR models. The observed emission is taken to be the sum of the input power law and the outward emitted spectrum.

We ran models with radii from $10^{8} \mathrm{~cm}$ (the expected radius of the inner disk) to $10^{12} \mathrm{~cm}$ (a few times the size of the binary system during outburst, which is $\left.R \sim R_{*} \sim 5 R_{\odot}\right)$. Most of the reprocessing column should be contained within this radius. Close to the neutron star, the accretion flow can create large gas densities and columns $\sim 10^{24} \mathrm{~cm}^{-2}$ or greater. At $R \gtrsim R_{*}$, the wind from the Be star is expected to blow away the surrounding interstellar medium so that the gas density is determined mainly by the star's mass loss. For a spherical wind,

$$
\dot{M}=n \mu m_{\mathrm{H}} 4 \pi R^{2} v_{w},
$$

where $\mu$ is the molecular weight, $\sim 1.4$. For $\dot{M} \lesssim 10^{-6} M_{\odot} \mathrm{yr}^{-1}$ and $v_{w} \gtrsim 1000 \mathrm{~km} \mathrm{~s}^{-1}$, we have upper limits in our models of $N \lesssim 10^{23.5}$ and $10^{23} \mathrm{~cm}^{-2}$ for $R=10^{11.5}$ and $10^{12} \mathrm{~cm}$, respectively. We note that, while large densities may be possible at smaller radii, we did not run models with $N>10^{24} \mathrm{~cm}^{-2}$ because the effects of Compton scattering are not explicitly included in XSTAR. We discuss Compton-thick reprocessing in the next section.

In most models, we allowed XSTAR to vary the temperature in each subshell to minimize heating and/or cooling as it stepped outward through the cloud. However, for some runs at large radii $\left(10^{11.5}-10^{12} \mathrm{~cm}\right)$, the model encountered problems converging on the temperature. In these cases we set the entire cloud to be at the single temperature that minimized heating and/or cooling over the entire cloud.

\subsection{XSTAR Results}

Outputs of sample model runs are given in Table 4. Because a simple power law fits the hard end of the spectrum well, there is likely to be little absorbing gas in the system directly along the line of sight, so the observed spectrum is the sum of the input hard power law plus the reprocessed emission from surrounding gas. Samples of these spectra are shown in Figure 7.

We find that none of the models are able to reproduce the observed emission from RX J0059.2-7138. In models in which $\log \xi \gtrsim 5$ (that is, for small radii) and there is virtually no reprocessing for any column density, because the gas temperature is very high $\left(\sim 5 \times 10^{7} \mathrm{~K}\right)$, all the atoms in the gas are completely ionized, and therefore, the opacity to photoionziation interactions is low. Reprocessing inside this radius requires 
TABLE 4

Results of Selected XSTAR Runs

\begin{tabular}{|c|c|c|c|c|c|c|c|c|c|c|}
\hline \multirow[b]{2}{*}{ RuN } & \multirow[b]{2}{*}{$\log R$} & \multirow[b]{2}{*}{$\log N$} & \multirow[b]{2}{*}{$\log n$} & \multirow[b]{2}{*}{$\log \xi$} & \multicolumn{2}{|c|}{$Z=0.25$} & \multicolumn{2}{|c|}{$Z=0.01$} & \multicolumn{2}{|c|}{$Z=1$} \\
\hline & & & & & $\log T$ & $f_{\text {rep }}$ & $\log T$ & $f_{\text {rep }}$ & $\log T$ & $f_{\text {rep }}$ \\
\hline \multicolumn{11}{|c|}{$\Delta R=R$} \\
\hline $1 \ldots$ & 9.0 & 24.0 & 15.0 & $4.7-5.4$ & $7.6-7.6$ & $1.0 \times 10^{-4}$ & $7.6-7.6$ & $1.3 \times 10^{-5}$ & $7.6-7.6$ & $3.8 \times 10^{-4}$ \\
\hline $2 \ldots \ldots \ldots \ldots \ldots . .$. & 10.0 & 24.0 & 14.0 & $3.7-4.4$ & $6.9-7.5$ & $3.6 \times 10^{-3}$ & $7.0-7.5$ & $4.9 \times 10^{-4}$ & $6.9-7.5$ & 0.014 \\
\hline 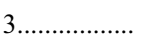 & 11.0 & 23.5 & 12.5 & $3.2-3.9$ & $4.6-7.2$ & 0.045 & $4.6-7.2$ & 0.099 & $6.4-7.2$ & 0.018 \\
\hline 4....................... & 11.0 & 24.0 & 13.0 & $2.7-3.4$ & $4.7-6.5$ & 0.14 & $4.5-5.6$ & 0.022 & $6.2-6.6$ & 0.14 \\
\hline $5 \ldots \ldots \ldots \ldots \ldots . . .$. & 11.5 & 23.5 & 12.0 & $2.7-3.4$ & $4.6-6.4$ & 0.087 & $4.3-5.1$ & 0.040 & $5.2-6.6$ & 0.099 \\
\hline $6 \ldots \ldots \ldots \ldots \ldots .$. & 12.0 & 23.0 & 11.0 & $2.7-3.4$ & $5.4-6.5$ & $8.7 \times 10^{-3}$ & $4.8^{\mathrm{a}}$ & $7.7 \times 10^{-3}$ & $6.2-6.6$ & 0.022 \\
\hline \multicolumn{11}{|c|}{$\Delta R=0.1 R$} \\
\hline 7.................... & 11.0 & 24.0 & 14.0 & $2.3-2.4$ & $4.5-5.1$ & 0.21 & $4.5-4.9$ & 0.045 & $4.4-5.8$ & 0.41 \\
\hline 8 & 11.5 & 23.5 & 13.0 & $2.3-2.4$ & $4.8^{\mathrm{a}}$ & 0.14 & $4.7^{\mathrm{a}}$ & 0.044 & $4.6^{\mathrm{a}}$ & 0.26 \\
\hline 9 & 12.0 & 23.0 & 13.0 & $2.3-2.4$ & $4.8^{\mathrm{a}}$ & 0.061 & $4.5^{\mathrm{a}}$ & 0.051 & $4.6^{\mathrm{a}}$ & 0.14 \\
\hline
\end{tabular}

NotE.-All quantities are in cgs units.

${ }^{a}$ Model run with constant temperature throughout cloud.

densities much higher than those that are possible to model using XSTAR. This approaches dense, optically thick reprocessing, which is discussed in the next section.

For larger $R \geq 10^{11} \mathrm{~cm}$ (and thus smaller $\xi$ ), the spectrum is markedly altered by the photoionized gas, as shown in Figure 7. Some of the hard X-rays are absorbed through ionization and reemitted as softer photons through recombination, bremsstrahlung, or line emission. For each model, we calculate $f_{\text {rep}}$, the ratio of the total luminosity emitted outward by the gas to the total input luminosity. For most models $f_{\text {rep }}<0.1$, and at most $50 \%$ of the reprocessed emission emerges in soft X-rays $(0.1-2 \mathrm{keV})$. There are a few cases for the $\Delta R=0.1 R$ shells that have $f_{\text {rep }}>0.2$, but in these only $10 \%-20 \%$ of the emission emerges in soft X-rays, with most coming in the EUV. In addition, the spectral shape of the soft emission does not match observations. There is little bremsstrahlung continuum, and the

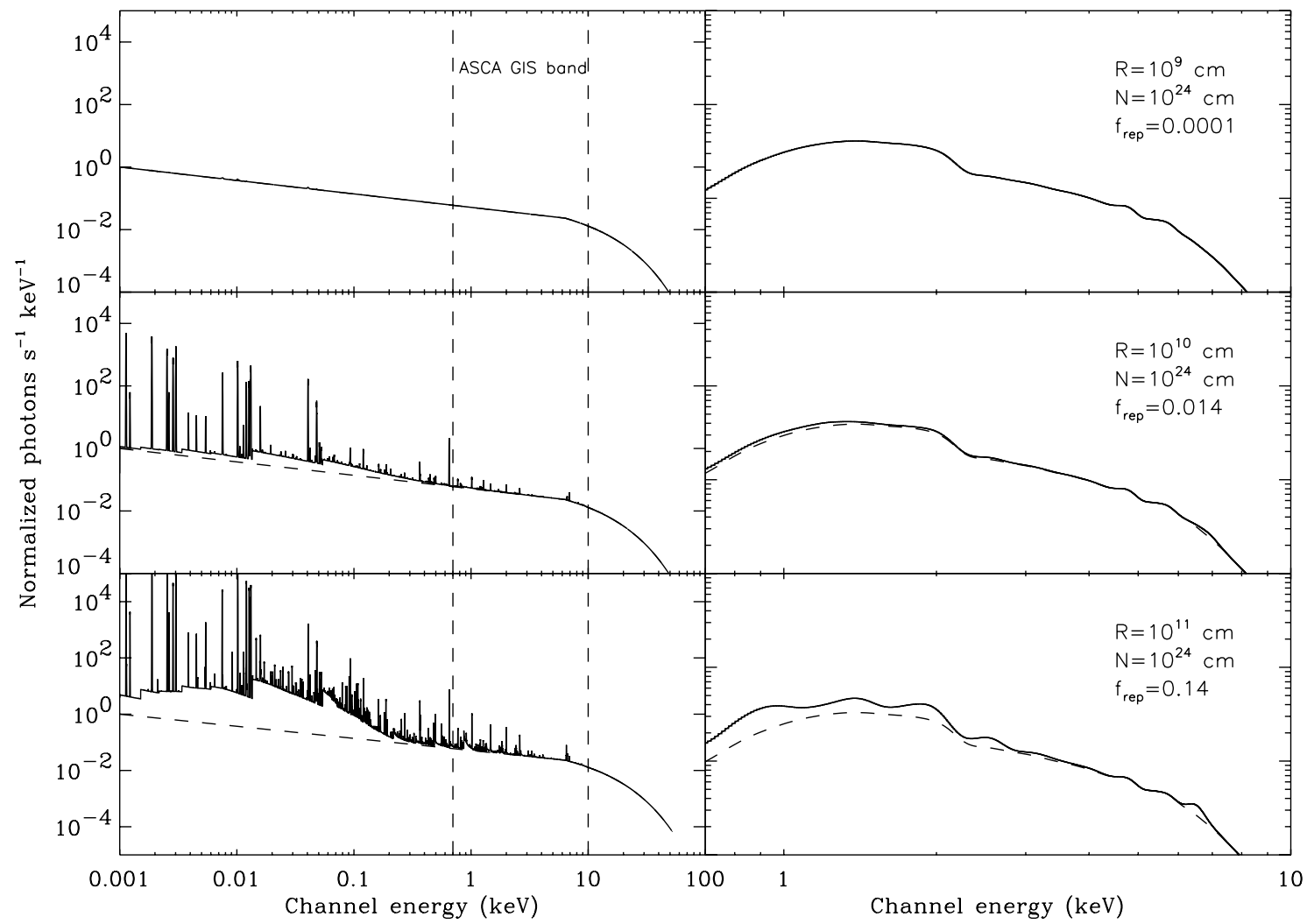

FIG. 7.-Left: Sample photon spectra from XSTAR, showing the effects of increased reprocessing with larger radius. The models have metallicity $Z=0.25 Z_{\odot}$, $N=10^{24} \mathrm{~cm}^{-2}$, and $\Delta R=R$. The dotted line shows the input spectrum, matching the exponentially cutoff power law for RX J0059.2-7138. Note the recombination continuum at energies below $0.1 \mathrm{keV}$. Right: Part of each spectrum for $0.7-10 \mathrm{keV}$, convolved with the response of the $A S C A$ GIS 2 detector. Note that features become visible in the $A S C A$ spectrum as reprocessing increases. 
radiative recombination and atomic lines produce spectra with discrete features and insufficient flux at $\sim 1 \mathrm{keV}$.

To show this quantitatively, we used the output XSTAR spectra to create table models in XSPEC and fitted these models to the observed spectrum of RX J0059.2-7138. We took data from the ASCA GIS2 observation of 1993 May (Kohno et al. 2000) from the HEASARC archive. We used the standard event-screening criteria and extracted the source and background spectra from regions similar to those shown in Figure 1 of Kohno et al. (2000). We fitted the data in the range 0.7$7 \mathrm{keV}$.

For each XSTAR run, the model consisted of the input power-law spectrum plus the outward emitted spectrum, modified by typical interstellar $N_{\mathrm{H}}=4 \times 10^{20} \mathrm{~cm}^{-2}$. We fitted these models to the data, allowing the relative luminosities of the input and emitted component to vary between 0.01 and 100 times that calculated by XSTAR (indicating more or less reprocessing gas than in our model shells). The models fitted poorly, all having $\chi_{\nu}^{2} \geq 2.5$ and most having $\chi_{\nu}^{2}>7$. In general, the reprocessed spectra have discrete line features and too little flux at $\sim 1 \mathrm{keV}$. In addition, most spectra are underluminous; for the model with the best fit $(Z=0.01, R=$ $10^{12} \mathrm{~cm}, N=10^{23} \mathrm{~cm}^{-2}$, and $\Delta R=0.1 R$ ), the condition $\chi_{\nu}^{2}=$ 2.5 requires an outward luminosity 90 times that output by XSTAR.

To allow for reprocessing by gas at several different radii and densities, we also performed spectral fits with multiple models simultaneously. For each $Z$, we included the emitted spectra from the six models with the most luminous emission in soft $\mathrm{X}$-rays $(0.1-2 \mathrm{keV})$ plus the input power law. We allowed the normalization of each emitted spectrum and the input power law to vary, and the results are shown in Figure 8. Even when combining emission from several sets of gas parameters, there remains a decrement at low energies, which is not significantly affected by changing $N_{\mathrm{H}}$. As shown in $\S 4$, this required soft flux could not simply be part of the emission from the neutron star because of the high temperatures required.

Of course, the photoionized gas in XBP systems is far more complex than we have modeled here, containing shocks, wakes, and other density gradients, and in the case of Be stars, a circumstellar disk. We have not considered small regions of very dense gas $\left(n>10^{12} \mathrm{~cm}^{-3}\right)$ at large radii, such as those present in simulations of SMC X-1 (Blondin \& Woo 1995) and suggested by the lines seen by Vrtilek et al. (2001). We note, however, that such dense regions subtend a small angle to the neutron star and so do not contribute significantly to the reprocessed flux. There also could be variations in the shape of the photoionizing spectrum seen by different parcels of gas, which would affect the photoionization emission in complex ways that would require a much more detailed study to model completely. Thus, it is in principle possible that a particular configuration might produce the soft spectrum seen in RX J0059.2-7138. However, our analysis suggests that in general photoionized emission is too weak and would have an incorrect spectral shape. In RX J0059.2-7138, as in the other luminous XBPs, the soft excess most likely has a different origin.

\subsection{Higher Optical Depths}

Because XSTAR does not include Comptonization, our models have not covered the case of Compton-thick $\left(N>10^{24} \mathrm{~cm}^{-2}\right)$ gas at radii close to the neutron star, where high densities may be present. Ross (1979) models this regime with code that explicitly calculates the radiative transfer in Compton-thick gas.
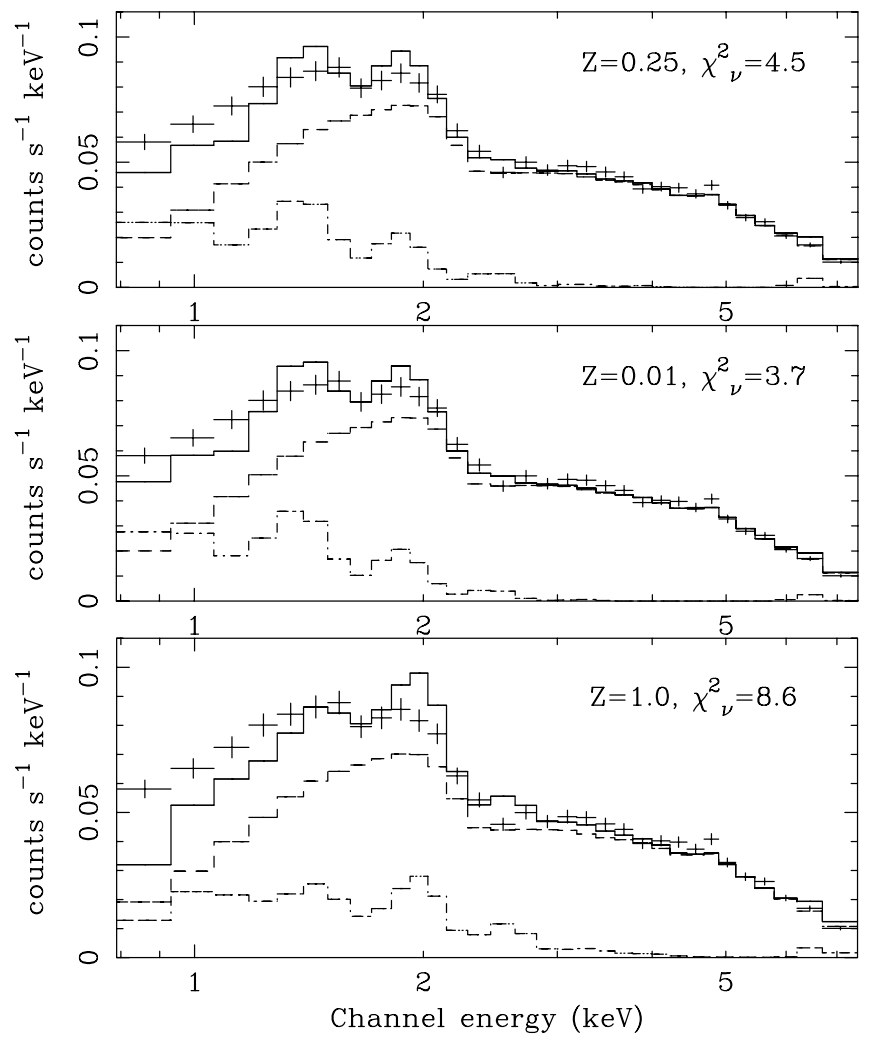

FIG. 8.-Observed ASCA GIS2 spectrum for RX J0059.2-7138, fitted with the input power law plus the six most luminous outward emission spectra from XSTAR, for $Z=0.25,0.01$, and $1 Z_{\odot}$. Each fit had 24 degrees of freedom, and the best-fit $\chi_{\nu}^{2}$ values are as shown. The dashed lines indicate the contributions from the power law and the reprocessed emission. Note that even when emission from multiple models is included, the spectra do not reproduce the observations.

The models show that for $R \sim 10^{8} \mathrm{~cm}$, high densities $(n \sim$ $10^{18} \mathrm{~cm}^{-3}$ ), and $L_{\mathrm{X}}=2 \times 10^{37} \mathrm{ergs} \mathrm{s} \mathrm{s}^{-1}$, there is still some decrement from a blackbody shape at X-ray energies, even for high Thomson optical depths $\tau_{\mathrm{T}} \sim 3-5$. In this regime there are strong emission features of oxygen and silicon at $\sim 0.8-$ $1.2 \mathrm{keV}$, which are not seen in the soft excess of most XBPs but may correspond to the $\sim 1 \mathrm{keV}$ emission lines found in Her $\mathrm{X}-1$. This suggests that close into the neutron star, where we expect high gas densities, the gas must have high Thomson optical depths to produce the blackbody-shaped spectrum of the soft excess. We discuss this regime in the next section.

\section{REPROCESSING BY OPTICALLY THICK MATERIAL}

Reprocessing might occur in gas that is completely optically thick, with $\tau_{\mathrm{T}}>5$. Such material is naturally expected to reside in the accretion disk, for example, and when illuminated with hard X-rays it reradiates in a blackbody-like spectrum. If the surface area of this region is of the appropriate size, it can reproduce the observed temperatures and luminosities. The exact geometry of the accreting material is unknown, but for simplicity we use the phrase "reprocessing by the inner disk" to refer to all shapes (shells, warped surfaces, etc.) of optically thick gas at the disk's inner edge.

To begin we consider a thick partial spherical shell centered on the neutron star, subtending a solid angle $\Omega$ at the X-ray source (see Fig. 9). Any photons that strike this gas are absorbed and reprocessed, so that for isotropic emission,

$$
L_{\mathrm{soft}}=\Omega L_{\mathrm{X}} .
$$




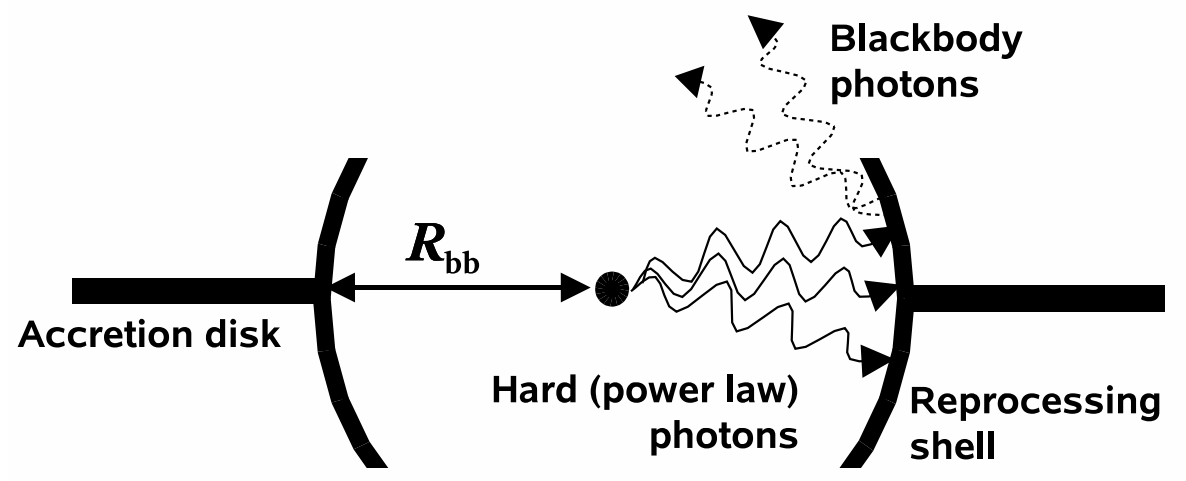

(solid angle $\Omega$ )

FIG. 9.- Simple picture used to calculate the blackbody emission radius $R_{\mathrm{BB}}$.

If the radius of this shell is $R_{\mathrm{BB}}$, the reprocessing region has area

$$
A=\Omega 4 \pi R_{\mathrm{BB}}^{2}
$$

For isotropic blackbody radiation,

$$
L_{\mathrm{soft}}=\Omega 4 \pi R_{\mathrm{BB}}^{2} \sigma T_{\mathrm{BB}}^{4}
$$

Thus, we have

$$
R_{\mathrm{BB}}^{2}=\frac{L_{\mathrm{X}}}{4 \pi \sigma T_{\mathrm{BB}}^{4}} .
$$

This differs from some estimates for $R_{\mathrm{BB}}$, which assume all the soft luminosity is radiated from a full sphere at $R_{\mathrm{BB}}$. Our formula is more appropriate if the blackbody comes from reprocessing by a partially covering disklike structure. For typical $L_{\mathrm{X}}=2 \times 10^{38} \mathrm{ergs} \mathrm{s}^{-1}$ and $k T_{\mathrm{BB}}=0.17 \mathrm{keV}$, we obtain $R_{\mathrm{BB}}=10^{8} \mathrm{~cm}$. This value is close to the typical radius of the magnetosphere for the luminous XBPs, suggesting that the reprocessing gas may be at the inner edge of the accretion disk.

\subsection{Her $X-1$ and Cen $X-3$}

There is evidence for the above picture in Her X-1 and Cen $\mathrm{X}-3$. In Her $\mathrm{X}-1$, the 35 day periodicity is attributed to the blocking of the central neutron star by a precessing disk (e.g., Gerend \& Boynton 1976). Detailed geometric models for the disk have been used to explain phenomena such as X-ray dips (Shakura et al. 1999) and varying pulse profiles (Scott et al. 2000). The anomalous low states observed in 1983, 1993, and 1999 in which the source remained in the low state for several 35 day periods have also convincingly been shown to be due to occultation by the disk (Coburn et al. 2000; Manchanda 2003).

Observations suggest that this blocking disk reprocesses the hard X-rays to produce the soft excess. Endo et al. (2000) performed pulse-phase resolved spectroscopy on $A S C A$ data taken during the main-on state $\left(\phi_{35} \sim 0.1\right)$. As described in $\S 2$, they found that the soft blackbody component and line emission features pulsed $230^{\circ}$ out of phase from the narrow power-law pulses, an offset seen earlier by McCray et al. (1982). The authors proposed that the tilted inner accretion disk absorbs the hard X-rays as the pulsar points away from the line of sight, giving rise to the blackbody and lines, which are emitted back toward the observer out of phase with the hard pulses. For the spectral parameters found by Endo et al. (2000), $R_{\mathrm{BB}} \sim 5 \times$ $10^{7} \mathrm{~cm}$. They showed that the cooling timescale of the irradiated gas is a small fraction of a second, so it is possible for the reprocessed soft X-rays to pulse along with the hard component.

Ramsay et al. (2002) analyzed observations with $X M M$ taken at $\phi_{35}=0.17$ (close to main-on), 0.26 (low state), and 0.60 (short-on state). They examined how the phase shift between the soft excess and hard component varies with $\phi_{35}$. They found that the profiles have phase offsets that vary from $150^{\circ}$ at $\phi_{35}=0.17$ to $-10^{\circ}$ at $\phi_{35}=0.26$ and $90^{\circ}$ at $\phi_{35}=0.6$. They argued that this continuous change in offset is expected from reprocessing by a tilted precessing disk.

For Her X-1, the range of observed values $k T_{\mathrm{BB}} \sim 0.1-$ $0.16 \mathrm{keV}$ and $L_{\mathrm{X}} \sim 2 \times 10^{37}$ ergs $\mathrm{s}^{-1}$ gives $R_{\mathrm{BB}} \sim 0.5-1.2 \times$ $10^{8} \mathrm{~cm}$. An estimate of the magnetospheric radius (Frank et al. 2002) is

$$
R_{m} \sim 0.5 R_{\mathrm{A}} \sim 1.5 \times 10^{8} m_{1}^{1 / 7} R_{6}^{10 / 7} L_{37}^{-2 / 7} B_{12}^{4 / 7} \mathrm{~cm}
$$

where $R_{\mathrm{A}}$ is the standard Alfvén radius. Here $m_{1}$ is the mass of the neutron star in $M_{\odot}, R_{6}$ is its radius in $10^{6} \mathrm{~cm}, L_{37}$ is the X-ray luminosity in $10^{37} \mathrm{ergs} \mathrm{s}^{-1}$, and $B_{12}$ is the neutron star surface magnetic field in $10^{12} \mathrm{G}$. For Her X-1 $\left(R_{6} \sim 1\right.$ and $B_{12} \sim$ 4), $R_{m} \sim 3 \times 10^{8} \mathrm{~cm}$. Scott et al. (2000) calculate $R_{m}$ using several other models (i.e., Finger et al. 1996; Kiraly \& Meszaros 1988; Aly 1980) and find they all give $R_{m} \sim(3-4) \times 10^{8} \mathrm{~cm}$. Thus, $R_{m}$ is larger than $R_{\mathrm{BB}}$ by as much as a factor of 4 or more. We discuss possible explanations for this discrepancy in the next subsection.

In Cen X-3, the soft excess is less well studied but is again consistent with reprocessing by the disk. Burderi et al. (2000) found a bright soft excess in the BeppoSAX spectrum, with $k T_{\mathrm{BB}}=0.11 \mathrm{keV}$ and containing $58 \%$ of the total unabsorbed flux. With $L_{\mathrm{X}} \sim 10^{38} \operatorname{ergs~s}{ }^{-1}$, we have $R_{\mathrm{BB}} \sim 2.4 \times 10^{8} \mathrm{~cm}$, while $B_{12} \sim 3$ gives $R_{m} \sim 1.5 \times 10^{8} \mathrm{~cm}$. As in Her X-1, the blackbody and iron line are likely produced by reprocessing at the magnetosphere. Kohmura et al. (2001) tested this picture by examining aperiodic oscillations of the X-ray flux and measuring the time delay of the iron lines with respect to the other bands. They found a systematic time delay of $0.39 \pm 1.0 \mathrm{~ms}$, which implies a light-travel distance of $\sim 1.7 \times 10^{8} \mathrm{~cm}$. This 
distance is comparable to $R_{\mathrm{BB}}$ and $R_{m}$ and so supports the interpretation of disk reprocessing.

\subsection{SMC $X-1$ and $L M C X-4$}

Although SMC X-1 and LMC X-4 are more luminous than Her X-1, they all show remarkable similarities including a sinusoidal soft pulse profile and a long-term periodicity, which is also attributed to a blocking, precessing disk. These similarities indicate that optically thick reprocessing may be the source of the soft excess in these systems as well.

Given the temperatures and luminosities for SMC X-1 and LMC X-4 from Paul et al. (2002; see Table 1), we find $R_{\mathrm{BB}}=$ 1.3 and $1.1 \times 10^{8} \mathrm{~cm}$, respectively. For LMC X-4, $B_{12} \sim 10$ indicates $R_{m} \sim 2.9 \times 10^{8} \mathrm{~cm}$. This gives $R_{\mathrm{BB}} / R_{m}=0.4$, compared with $R_{\mathrm{BB}} / R_{m} \sim 0.2-0.4$ for Her $\mathrm{X}-1$, depending on the value for $T_{\mathrm{BB}}$, and $\sim 1.5$ for Cen $\mathrm{X}-3$. The values for $R_{\mathrm{BB}}$ and $R_{m}$ are shown for comparison in Table 5.

In SMC X-1, no cyclotron feature has been detected, so there is no magnetic field estimate. For SMC X-1 to have $R_{\mathrm{BB}} / R_{m}=0.4$, as in Her $\mathrm{X}-1$ and LMC X-4, would require $B_{12} \sim 20$. Such a high magnetic field would give a cyclotron line at $\sim 200 \mathrm{keV}$ and so may be difficult to detect with current instruments. However, Makishima et al. (1999) suggest that the relatively low spectral cutoff energy (6 keV) in SMC X-1 may indicate a smaller magnetic field than Her X-1, so the question is still open. Another estimate for the inner edge of the accretion disk is the corotation radius

$$
R_{\mathrm{cor}}=\left(G M / \Omega_{\mathrm{NS}}^{2}\right)^{1 / 3},
$$

which is the radius at which the Keplerian angular velocity of the disk is equal to $\Omega_{\mathrm{NS}}$, the angular velocity of the neutron star. For SMC X-1, $R_{\text {cor }}=1.3 \times 10^{8} \mathrm{~cm}$, which is equal to $R_{\mathrm{BB}}$.

Paul et al. (2002) argue that because of discrepancies between $R_{\mathrm{BB}}$ and $R_{m}$, disk reprocessing may not be at work in the luminous XBPs. However, Her X-1 and Cen X-3 show substantial evidence for reprocessing by the disk, yet have markedly different ratios between $R_{\mathrm{BB}}$ and $R_{m}$. It is therefore difficult to base conclusions on the exact values obtained for $R_{\mathrm{BB}}$ and $R_{m}$. This uncertainty exists for several reasons, some of which are treated in detail by Scott et al. (2000). First, there may be errors in our estimate of $R_{m}$, largely because the standard models for the magnetospheric radius are incomplete. The interaction of the accretion disk with the magnetic field is poorly understood, but certainly a high rate of mass flow from the disk onto the magnetic field lines has some effect on the field (e.g., Agapitou \& Papaloizou 2000; Aly \& Kuijpers 1990). Whether this makes $R_{m}$ larger or smaller is uncertain. It may also be that the inner edge of the disk does not actually reside at the radius that we define as the magnetosphere but that some gas exists inside this radius. Again, such a process is not fully understood and warrants more investigation.

Even if the standard formulae are accurate and the accretion disk really is truncated at $R_{m}$, there are uncertainties in the parameters that are used to calculate $R_{m} . B$ is taken from measurements of a cyclotron feature that may arise not in the main dipole field but in a region of stronger or weaker field near the accretion flow. Since $R_{m} \propto B^{4 / 7}$, a $25 \%$ error in the $B$ field strength would cause a $\sim 15 \%$ error in $R_{m}$. There is also uncertainty in $L_{\mathrm{X}}$ due to errors in the distance to the source, uncertainty in the spectral parameters, and variation of the source with time. Since $R_{m} \propto L_{\mathrm{X}}^{-2 / 7}$, a $25 \%$ error in $L_{\mathrm{X}}$ would cause a $\sim 7 \%$ error in $R_{m}$. There are also uncertainties in the masses and radii of neutron stars. Masses of solitary pulsars seem to be
TABLE 5

Blackbody and Magnetospheric Radil for Selected XBPs

\begin{tabular}{ccccc}
\hline \hline Parameter & Her X-1 & Cen X-3 & LMC X-4 & Vela X-1 \\
\hline$L_{\mathrm{X}}\left(10^{38} \mathrm{ergs} \mathrm{s}^{-1}\right) \ldots \ldots \ldots$ & 0.2 & 1.0 & 1.2 & 0.01 \\
$B\left(10^{12} \mathrm{G}\right) \ldots \ldots \ldots \ldots \ldots \ldots$ & 4 & 3.5 & 10 & 2.4 \\
$k T_{\mathrm{BB}}(\mathrm{keV}) \ldots \ldots \ldots \ldots \ldots .$. & $0.16,0.1$ & 0.11 & 0.17 & 0.2 \\
$R_{\mathrm{BB}}\left(10^{8} \mathrm{~cm}\right) \ldots \ldots \ldots \ldots \ldots$. & $0.49,1.2$ & 2.3 & 1.1 & 0.07 \\
$R_{m}\left(10^{8} \mathrm{~cm}\right) \ldots \ldots \ldots \ldots \ldots$. & 2.9 & 1.5 & 2.9 & 4.5 \\
$R_{\mathrm{BB}} / R_{m} \ldots \ldots \ldots \ldots \ldots \ldots .$. & $0.2,0.4$ & 1.5 & 0.4 & 0.01 \\
\hline
\end{tabular}

tightly peaked around $1.4 M_{\odot}$ (Thorsett \& Chakrabarty 1999), but for accreting pulsars the masses may be greater. For example, from radial velocities of the companion to Vela X-1, Quaintrell et al. (2003) obtain $M=2.27 \pm 0.17 M_{\odot}$. Since $R_{m} \propto M^{1 / 7}$, such a mass variation would correspond to an increase in $R_{m}$ of $\sim 10 \%$. Estimates of $R_{\mathrm{NS}}$ vary from 0.7 to $1.6 \times 10^{6} \mathrm{~cm}$, which can vary $R_{m}$ by another $\sim 20 \%$. The overall effect of these uncertainties is to create substantial uncertainty in $R_{m}$, which may be as high as $\sim 30 \%$ or greater.

There may also be errors in our estimate of $R_{\mathrm{BB}}$. The spectrum of the reprocessing material is almost certainly not a single-temperature blackbody. A hot disk corona could Compton-scatter some of the blackbody photons, giving an observed $T$ higher than the true blackbody temperature and causing an underestimate of $R_{\mathrm{BB}}$. On the other hand, the reprocessing surface may be tilted with respect to the hard X-ray flux, which would increase the emitting area at a given radius and thus decrease $R_{\mathrm{BB}}$. Viewing angle effects due to nonisotropic emission could also be important.

Given these uncertainties, the correspondence between $R_{m}$ and $R_{\mathrm{BB}}$ to a factor of 2 or so suggests that reprocessing by the inner disk can explain the spectra of LMC X-4 and SMC X-1. Such a model arises naturally from the geometry of these sources, and, unlike the other processes we have considered, it reproduces the pulsations and high luminosities of the soft component.

\section{3. $R X$ J0059.2-7138 and XTE J0111.2-7317}

Transient $\mathrm{Be} / \mathrm{XBP}$ systems are expected to form accretion disks (Hayasaki \& Okazaki 2004), so inner disk reprocessing might occur. In RX J0059.2-7138 and XTE J0111.2-7317, there is no magnetic field measurement, so estimates of $R_{m}$ are difficult. A typical value of $B$ for XBPs $\left(B_{12}=4\right)$ gives $R_{m} \sim 1.5 \times 10^{8} \mathrm{~cm}$ for XTE J0111.2-7317. This is of order the $R_{\mathrm{BB}} \sim 0.8 \times 10^{8} \mathrm{~cm}$ obtained from the $A S C A$ spectrum (Yokogawa et al. 2000). RX J0059.2-7138 has a similar luminosity and spectrum and so may also have disk reprocessing. As in SMC X-1 and LMC X-4, this is the only mechanism that can produce the spectral shape and high $L_{\text {soft }}$ in these systems.

A puzzle is the lack of pulsation in the soft component of RX J0059.2-7138. One explanation is that, unlike Her X-1, SMC X-1, and LMC X-4, the disk may be at low inclination. In this case, the whole reprocessing region would present roughly the same angle to the observer, so we would see a brightly emitting disk at all phases in the pulsation. Another possibility is that electron scattering from a large disk wind or corona, of size $\sim 10^{10} \mathrm{~cm}$, could wash out the pulsations in the soft component.

\subsection{Vela $X-1$ and $4 U 1626-67$}

In Vela $\mathrm{X}-1$, reprocessing by the disk is not the main source of the soft excess. Given $L_{\mathrm{X}} \sim 10^{36} \mathrm{ergs} \mathrm{s}^{-1}$ and $B_{12} \sim 2.4$, we obtain $R_{m} \sim 5 \times 10^{8} \mathrm{~cm}$. Although a blackbody fit has not 
been performed to the Vela X-1 soft component, we note that Paul et al. (2002) were able to fit both blackbody and bremsstrahlung models to SMC X-1 and LMC X-4 spectra, giving $k T_{\mathrm{BB}}=0.17$ and $0.18 \mathrm{keV}$, and $k T_{\mathrm{tb}}=0.3$ and $0.5 \mathrm{keV}$, respectively. For Vela $\mathrm{X}-1, k T_{\mathrm{tb}}=0.5 \mathrm{keV}$, so we assume an equivalent $k T_{\mathrm{BB}} \sim 0.2 \mathrm{keV}$. This gives $R_{\mathrm{BB}} \sim 7 \times 10^{6} \mathrm{~cm}$, or $R_{\mathrm{BB}} / R_{m} \sim 0.01$, which is extremely small. In other words, Vela X-1 is not luminous enough to produce a reprocessed blackbody soft component at $k T_{\mathrm{BB}} \sim 0.2 \mathrm{keV}$ and over an area $\sim R_{m}^{2}$. On the other hand, because of the relatively low luminosity, the soft component is well explained by emission from diffuse gas excited by collisions and photoionization, as discussed in $\S \S 5$ and 6 , respectively.

The situation in $4 \mathrm{U} 1626-67$ is difficult to evaluate because the distance to the source, and thus $L_{\mathrm{X}}$, is poorly constrained. The different possibilities for a Roche lobe-filling companion to $4 \mathrm{U} 1626-67$ imply different distances: $1 \mathrm{kpc}$ for a $0.02 M_{\odot}$ white dwarf, $3 \mathrm{kpc}$ for a $0.08 M_{\odot}$ partially degenerate, hydrogen-depleted star, and $36 \mathrm{kpc}$ for a $0.6 M_{\odot}$ heliumburning star (Chakrabarty 1998). Chakrabarty (1998) found that optical photometry is consistent with emission coming mostly from an X-ray-heated disk, which implies a distance in the range $5 \mathrm{kpc} \lesssim D \lesssim 13 \mathrm{kpc}$.

Orlandini et al. (1998a) find that for a blackbody soft excess, the radius of the emitting region is $1.5 D_{\mathrm{kpc}} \mathrm{km}$. For $D \sim$ $5 \mathrm{kpc}$ and thus $L_{\mathrm{X}} \sim 2 \times 10^{36} \mathrm{ergs} \mathrm{s}^{-1}$, this region is the size of the neutron star, so soft excess may be thermal emission from the star's surface. To evaluate the possibility of reprocessing by the disk, we take $B_{12}=3.5, k T_{\mathrm{BB}}=0.3 \mathrm{keV}$, and $L_{\mathrm{X}}=$ $7.7 \times 10^{34} D_{\mathrm{kpc}}^{2}$ and find that $R_{\mathrm{BB}}$ is of order $R_{m} \sim 9 \times 10^{7} \mathrm{~cm}$ only at $D \sim 100 \mathrm{kpc}$. This is an unphysically large distance. We conclude that disk reprocessing is not at work in this system.

\subsection{Pulsations from Disk Reprocessing}

To conclude this section, we show that soft pulses can arise from disk reprocessing in the luminous XBPs. Following the calculation of Endo et al. (2000), the total thermal energy of the soft emitting region is simply the volume of the region times its thermal energy density

$$
E_{\mathrm{BB}}=\Omega 4 \pi R_{\mathrm{BB}}^{2} d\left(\frac{3}{2} n k T_{\mathrm{BB}}\right),
$$

where $d$ is the penetration depth of hard X-rays into the heated region. We assume that most X-rays penetrate no more than a few Compton depths, so that $n d \sim 3 / \sigma_{\mathrm{T}} \sim 10^{25} \mathrm{~cm}^{-2}$. For typical $R_{\mathrm{BB}}=10^{8}, \Omega / 4 \pi \sim 0.1$, and $k T_{\mathrm{BB}}=0.17 \mathrm{~K}$, we have $E_{\mathrm{BB}} \sim 10^{32}$ ergs. The cooling timescale is simply $t_{\text {cool }}=$ $E_{\mathrm{BB}} / L_{\mathrm{soft}}$, which for $L_{\text {soft }} \sim 10^{37} \mathrm{ergs} \mathrm{s}^{-1}$ gives $t_{\text {cool }} \sim 10^{-5} \mathrm{~s}$. As in Her $\mathrm{X}-1$, this is much shorter than the pulsation period, so soft pulses should be possible for this reprocessing mechanism.

\section{DISCUSSION}

We have found that only a few of the models that have been proposed can successfully account for the observed soft excess in XBPs. In addition, the origin of the soft excess depends strongly on the luminosity of the source. For systems with high $L_{\mathrm{X}} \gtrsim 10^{38} \mathrm{ergs} \mathrm{s}^{-1}$ (SMC X-1, LMC X-4, RX J0059.2-7138, XTE J0111.2-7317, and Cen X-3), the soft excess can be explained only by reprocessing of the hard X-rays by optically thick accreting material. However, for systems with $L_{\mathrm{X}} \lesssim 10^{36} \mathrm{ergs} \mathrm{s}^{-1}$, the soft excess is emitted by diffuse gas through collisional heating or photoionization (Vela X-1, RX J0101.3-7211, and AX J0103-722) or is possibly thermal emission from the neutron star's surface (4U 1626-67 and X Per).

The more luminous XBPs probably also emit soft X-rays from a diffuse cloud and from the surface of the neutron star, but these components are dominated by the flux from the power law and from disk reprocessing. On the other hand, in lower $L_{\mathrm{X}}$ systems, we do not expect disk reprocessing to be important, since for blackbody emission at $R_{\mathrm{BB}}=R_{m}$, we have $T_{\mathrm{BB}} \propto L_{\mathrm{X}}^{1 / 4} / R_{m}^{1 / 2}$. Since $R_{m} \propto L_{\mathrm{X}}^{-2 / 7}$, this gives $T_{\mathrm{BB}} \propto$ $L_{\mathrm{X}}^{11 / 28}$. Thus, for lower $L_{\mathrm{X}}$ the temperature of the disk moves toward the EUV and out of the soft X-ray band.

For XBPs with moderate $L_{\mathrm{X}} \sim 10^{37} \mathrm{ergs} \mathrm{s}^{-1}$, it appears possible to have disk reprocessing (Her X-1), emission by diffuse gas (EXO 053109-6609.2), or possibly both (A053866 ). We thus have something of a continuum, in which the soft excess from more luminous XBPs comes primarily from disk reprocessing, while in less luminous ones it originates in optically thin emission or thermal emission from the neutron star's surface.

In addition to these general conclusions, this work raises a number of puzzling questions. One is the apparently high brightness temperature of the power-law component in SMC $\mathrm{X}-1$ ( $\S 4.1$ ). This may suggest that the accretion column is hotter or larger in extent than models predict. For Vela X-1, the question remains whether the soft excess emission is powered mainly by photoionization or collisional energy sources $(\S \S 5$ and 6). In RX J0059.2-7138, where disk reprocessing is likely at work, the complete absence of soft pulsations $(\S 7.3)$ is also worth further investigation.

It is important to examine the lack of exact correlation between $R_{\mathrm{BB}}$ and $R_{m}$ in the sources where we believe reprocessing by the accretion disk is at work. We have discussed a number of uncertainties in these calculations in $\S 7.2$, but this problem warrants further study. For example, a thorough investigation of nonblackbody effects in the disk reprocessing can help reduce uncertainty in $R_{\mathrm{BB}}$. Radiative transfer in an obliquely illuminated model disk has been treated by Psaltis (2002), and the structure and line emission of an illuminated disk atmosphere has been modeled in detail (Jimenez-Garate et al. 2001, 2002). Using such models to analyze the soft emission from XBPs can help diagnose the gas conditions near the disk's inner edge.

There remains the question of how the accreting material, which is expected to be in a geometrically thin disk, is able to subtend a substantial solid angle to the central neutron star. Interactions with the magnetic field are likely to be key in this process. For example, Spruit \& Taam (1990) have shown analytically that accreting matter can be tied to the magnetic field in equilibrium positions off the midplane of the disk, near its inner edge. Numerical three-dimensional magnetohydrodynamic simulations of accretion onto rotating magnetized stars (Romanova et al. 2003, 2002) show structures including funnel flows and dense "rings" that develop near $R_{m}$. These may be the sites of the X-ray reprocessing, and the soft excess emission may provide constraints on the properties of such structures.

We can also use the soft excess to study warping and precession of the disk, particularly in Her X-1, SMC X-1, and LMC X-4. A warping instability in a centrally illuminated disk was found theoretically by Pringle (1996) and has been explored in a number of studies (e.g., Wijers \& Pringle 1999; Lai 1999; Maloney \& Begelman 1997) that also predict the disk's 
precession. As Ramsay et al. (2002) have done in the case of Her X-1, it would be interesting to examine SMC X-1 and LMC X-4 and see how the reprocessing region on the disk moves as the disk precesses.

\section{SUMMARY}

In this paper we have reviewed a number of observations of the soft excess in X-ray pulsars and explored the origin of this feature. We find the following:

1. The soft excess is a very common, if not ubiquitous, feature of emission from XBPs. Observations of XBPs that have not shown a soft excess are most often limited by low flux, high absorbing column, or insufficient soft sensitivity.

2. For luminous $\left(L_{\mathrm{X}} \gtrsim 10^{38} \mathrm{ergs} \mathrm{s}^{-1}\right)$ sources, the soft components have high luminosities and soft spectral shapes that can be explained only by reprocessing of hard X-rays from the neutron star by optically thick accreting material, most likely near the inner edge of the accretion disk.

3. For less luminous $\left(L_{X} \lesssim 10^{36} \mathrm{ergs} \mathrm{s}^{-1}\right)$ sources, the soft excess is due to other processes, such as emission by photo- ionized or collisionally heated diffuse gas or thermal emission from the surface of the neutron star. In these sources, the soft component cannot come from reprocessing by the accretion disk.

4. XBPs of intermediate luminosity $\left(L_{\mathrm{X}} \sim 10^{37} \mathrm{ergs} \mathrm{s}^{-1}\right)$ show either or both of these types of emission.

5. Future observations of soft excess will allow us to explore aspects of XBPs, such as the properties of circumstellar gas, interaction of accreting matter and the magnetic field, and warping and precession of the accretion disk.

We thank Rosanne DiStefano, Craig Heinke, Joseph Neilsen, John Raymond, and Saku Vrtilek for helpful discussions, and the referee for useful comments. This work was supported by NSF grant AST 03-07433 and NASA grant NAG5-10780. The research has made use of NASA's Astrophysics Data System and of data obtained from the High Energy Astrophysics Science Archive Research Center (HEASARC), provided by NASA's Goddard Space Flight Center.
Agapitou, V., \& Papaloizou, J. C. B. 2000, MNRAS, 317, 273

Aly, J. J. 1980, A\&A, 86, 192

Aly, J. J., \& Kuijpers, J. 1990, A\&A, 227, 473

Angelini, L., White, N. E., Nagase, F., Kallman, T. R., Yoshida, A., Takeshima, T., Becker, C., \& Paerels, F. 1995, ApJ, 449, L41

Arnaud, K. A. 1996, in ASP Conf. Ser. 101, Astronomical Data Analysis Software and Systems V, ed. G. Jacoby \& J. Barnes (San Francisco: ASP), 17 Becker, P. A. 1998, ApJ, 498, 790

Bildsten, L., et al. 1997, ApJS, 113, 367

Blondin, J. M., \& Woo, J. W. 1995, ApJ, 445, 889

Boroson, B., Kallman, T., Blondin, J. M., \& Owen, M. P. 2001, ApJ, 550, 919

Boroson, B., Vrtilek, S. D., Kallman, T., \& Corcoran, M. 2003, ApJ, 592, 516

Burderi, L., Di Salvo, T., Robba, N. R., La Barbera, A., \& Guainazzi, M. 2000, ApJ, 530, 429

Burnard, D. J., Arons, J., \& Klein, R. I. 1991, ApJ, 367, 575

Chakrabarty, D. 1998, ApJ, 492, 342

Chakrabarty, D., Levine, A. M., Clark, G. W., \& Takeshima, T. 1998, IAU Circ. 7048

Choi, C. S., Dotani, T., Day, C. S. R., \& Nagase, F. 1996, ApJ, 471, 447

Clarkson, W. I., Charles, P. A., Coe, M. J., Laycock, S., Tout, M. D., \& Wilson, C. A. 2003, MNRAS, 339, 447

Coburn, W., Heindl, W. A., Gruber, D. E., Rothschild, R. E., Staubert, R., Wilms, J., \& Kreykenbohm, I. 2001, ApJ, 552, 738

Coburn, W., Heindl, W. A., Rothschild, R. E., Gruber, D. E., Kreykenbohm, I., Wilms, J., Kretschmar, P., \& Staubert, R. 2002, ApJ, 580, 394

Coburn, W., et al. 2000, ApJ, 543, 351

Coe, M. J., Haigh, N. J., \& Reig, P. 2000, MNRAS, 314, 290

Coe, M. J., Haigh, N. J., Wilson, C. A., \& Negueruela, I. 2003, MNRAS, 344, 1075

dal Fiume, D., et al. 1998, A\&A, 329, L41

di Salvo, T., Burderi, L., Robba, N. R., \& Guainazzi, M. 1998, ApJ, 509, 897

Endo, T., Ishida, M., Masai, K., Kunieda, H., Inoue, H., \& Nagase, F. 2002, ApJ, 574, 879

Endo, T., Nagase, F., \& Mihara, T. 2000, PASJ, 52, 223

Finger, M. H., Wilson, R. B., \& Harmon, B. A. 1996, ApJ, 459, 288

Forman, W., Jones, C., Tananbaum, H., Gursky, H., Kellogg, E., \& Giacconi, R. 1973, ApJ, 182, L103

Frank, J., King, A., \& Raine, D. J. 2002, Accretion Power in Astrophysics (3rd ed.; Cambridge: Cambridge Univ. Press)

Fransson, C., \& Fabian, A. C. 1980, A\&A, 87, 102

Gerend, D., \& Boynton, P. E. 1976, ApJ, 209, 562

Giacconi, R., Murray, S., Gursky, H., Kellogg, E., Schreier, E., \& Tananbaum, H. 1972, ApJ, 178, 281

Grindlay, J. E., Petro, L. D., \& McClintock, J. E. 1984, ApJ, 276, 621

Gruber, D. E., \& Rothschild, R. E. 1984, ApJ, 283, 546

Haberl, F. 1994, A\&A, 288, 791

Haberl, F., Aoki, T., \& Mavromatakis, F. 1994, A\&A, 288, 796

Haberl, F., Dennerl, K., \& Pietsch, W. 2003, A\&A, 406, 471

Hayasaki, K., \& Okazaki, A. T. 2004, MNRAS, 350, 971

Hughes, J. P. 1994, ApJ, 427, L25

\section{EFERENCES}

Jimenez-Garate, M. A., Raymond, J. C., \& Liedahl, D. A. 2002, ApJ, 581, 1297 Jimenez-Garate, M. A., Raymond, J. C., Liedahl, D. A., \& Hailey, C. J. 2001, ApJ, 558, 448

Jones, C., \& Forman, W. 1976, ApJ, 209, L131

Kallman, T., \& Bautista, M. 2001, ApJS, 133, 221

Kelley, R. L., Jernigan, J. G., Levine, A., Petro, L. D., \& Rappaport, S. 1983, ApJ, 264, 568

Király, P., \& Mészáros, P. 1988, ApJ, 333, 719

Kohmura, T., Kitamoto, S., \& Torii, K. 2001, ApJ, 562, 943

Kohno, M., Yokogawa, J., \& Koyama, K. 2000, PASJ, 52, 299

Kotani, T., Dotani, T., Nagase, F., Greenhill, J. G., Pravdo, S. H., \& Angelini, L. 1999, ApJ, 510, 369

Kretschmar, P., et al. 1996, A\&AS, 120, C175

Kreykenbohm, I., Coburn, W., Wilms, J., Kretschmar, P., Staubert, R., Heindl, W. A., \& Rothschild, R. E. 2002, A\&A, 395, 129

Krzeminski, W. 1974, ApJ, 192, L135

La Barbera, A., Burderi, L., Di Salvo, T., Iaria, R., \& Robba, N. R. 2001, ApJ, 553,375

Lai, D. 1999, ApJ, 524, 1030

Lang, F. L., et al. 1981, ApJ, 246, L21

Leong, C., Kellogg, E., Gursky, H., Tananbaum, H., \& Giacconi, R. 1971, ApJ, 170, L67

Levine, A., Rappaport, S., Putney, A., Corbet, R., \& Nagase, F. 1991, ApJ, 381,101

Li, F., Rappaport, S., \& Epstein, A. 1978, Nature, 271, 37

Liller, W. 1973, ApJ, 184, L37

Lucke, R., Yentis, D., Friedman, H., Fritz, G., \& Shulman, S. 1976, ApJ, 206, L25

Mahadevan, R., Narayan, R., \& Yi, I. 1996, ApJ, 465, 327

Makishima, K., Mihara, T., Nagase, F., \& Tanaka, Y. 1999, ApJ, 525, 978

Maloney, P. R., \& Begelman, M. C. 1997, ApJ, 491, L43

Manchanda, R. K. 2003, A\&A, 401, 1111

Mavromatakis, F., \& Haberl, F. 1993, A\&A, 274, 304

McClintock, J. E., Bradt, H. V., Doxsey, R. E., Jernigan, J. G., Canizares, C. R., \& Hiltner, W. A. 1977, Nature, 270, 320

McClintock, J. E., et al. 1976, ApJ, 206, L99

McCray, R. A., Shull, J. M., Boynton, P. E., Deeter, J. E., Holt, S. S., \& White, N. E. 1982, ApJ, 262, 301

Mereghetti, S., Bignami, G. F., Caraveo, P. A., \& Goldwurm, A. 1987, ApJ, 312,755

Mewe, R., Lemen, J. R., \& van den Oord, G. H. J. 1986, A\&AS, 65, 511

Middleditch, J., Mason, K. O., Nelson, J. E., \& White, N. E. 1981, ApJ, 244, 1001

Middleditch, J., \& Nelson, J. 1976, ApJ, 208, 567

Nagase, F. 1989, PASJ, 41, 1

2002, J. Astrophys. Astron., 23, 59

Nagase, F., Corbet, R. H. D., Day, C. S. R., Inoue, H., Takeshima, T., Yoshida, K., \& Mihara, T. 1992, ApJ, 396, 147

Naik, S., \& Paul, B. 2002, J. Astrophys. Astron., 23, 27 2004, ApJ, 600, 351 
Nishiuchi, M., et al. 1999, ApJ, 517, 436

Orlandini, M., dal Fiume, D., del Sordo, S., Frontera, F., Parmar, A. N., Santangelo, A., \& Segreto, A. 1999, A\&A, 349, L9

Orlandini, M., et al. 1998a, A\&A, 332, 121 . 1998b, ApJ, 500, L163

Owens, A., Oosterbroek, T., \& Parmar, A. N. 1997, A\&A, 324, L9

Paul, B., Nagase, F., Endo, T., Dotani, T., Yokogawa, J., \& Nishiuchi, M. 2002, ApJ, 579, 411

Petre, R., \& Gehrels, N. 1994, A\&A, 282, L33

Pfeffermann, E., \& Briel, U. G. 1986, Proc. SPIE, 597, 208

Pringle, J. E. 1996, MNRAS, 281, 357

Psaltis, D. 2002, ApJ, 574, 306

Quaintrell, H., Norton, A. J., Ash, T. D. C., Roche, P., Willems, B., Bedding, T. R., Baldry, I. K., \& Fender, R. P. 2003, A\&A, 401, 313

Ramsay, G., Zane, S., Jimenez-Garate, M. A., den Herder, J., \& Hailey, C. J. 2002, MNRAS, 337, 1185

Rappaport, S., Markert, T., Li, F. K., Clark, G. W., Jernigan, J. G., \& McClintock, J. E. 1977, ApJ, 217, L29

Roberts, M. S. E., Michelson, P. F., Leahy, D. A., Hall, T. A., Finley, J. P., Cominsky, L. R., \& Srinivasan, R. 2001, ApJ, 555, 967

Romanova, M. M., Ustyugova, G. V., Koldoba, A. V., \& Lovelace, R. V. E. 2002, ApJ, 578, 420

Romanova, M. M., Ustyugova, G. V., Koldoba, A. V., Wick, J. V., \& Lovelace, R. V. E. 2003, ApJ, 595, 1009

Ross, R. R. 1979, ApJ, 233, 334

Sadakane, K., Hirata, R., Jugaku, J., Kondo, Y., Matsuoka, M., Tanaka, Y., \& Hammerschlag-Hensberge, G. 1985, ApJ, 288, 284

Sako, M., Liedahl, D. A., Kahn, S. M., \& Paerels, F. 1999, ApJ, 525, 921

Sanduleak, N., \& Philips, A. G. D. 1977, IAU Circ. 3023

Santangelo, A., del Sordo, S., Segreto, A., dal Fiume, D., Orlandini, M., \& Piraino, S. 1998, A\&A, 340, L55

Sasaki, M., Pietsch, W., \& Haberl, F. 2003, A\&A, 403, 901

Schreier, E., Giacconi, R., Gursky, H., Kellogg, E., \& Tananbaum, H. 1972a, ApJ, 178, L71

Schreier, E., Levinson, R., Gursky, H., Kellogg, E., Tananbaum, H., \& Giacconi, R. 1972b, ApJ, 172, L79
Schulz, N. S., Canizares, C. R., Lee, J. C., \& Sako, M. 2002, ApJ, 564, L21 Schulz, N. S., Chakrabarty, D., Marshall, H. L., Canizares, C. R., Lee, J. C., \& Houck, J. 2001, ApJ, 563, 941

Scott, D. M., \& Leahy, D. A. 1999, ApJ, 510, 974

Scott, D. M., Leahy, D. A., \& Wilson, R. B. 2000, ApJ, 539, 392

Shakura, N. I., Prokhorov, M. E., Postnov, K. A., \& Ketsaris, N. A. 1999, A\&A, 348, 917

Shapiro, S. L., \& Teukolsky, S. A. 1983, Black Holes, White Dwarfs, and Neutron Stars: The Physics of Compact Objects (New York: Wiley)

Spruit, H. C., \& Taam, R. E. 1990, A\&A, 229, 475

Tananbaum, H., Gursky, H., Kellogg, E. M., Levinson, R., Schreier, E., \& Giacconi, R. 1972, ApJ, 174, L143

Tarter, C. B., Tucker, W. H., \& Salpeter, E. E. 1969, ApJ, 156, 943

Thorsett, S. E., \& Chakrabarty, D. 1999, ApJ, 512, 288

Vrtilek, S. D., Raymond, J. C., Boroson, B., Kallman, T., Quaintrell, H., \& McCray, R. 2001, ApJ, 563, L139

Webster, B. L., Martin, W. L., Feast, M. W., \& Andrews, P. J. 1972, Nature Phys. Sci., 240, 183

Welty, D. E. 2001, preprint (astro-ph/0110377)

White, N. E. 1978, Nature, 271, 38

Wijers, R. A. M. J., \& Pringle, J. E. 1999, MNRAS, 308, 207

Wilson, C. A., \& Finger, M. H. 1998, IAU Circ. 7048

Wojdowski, P., Clark, G. W., Levine, A. M., Woo, J. W., \& Zhang, S. N. 1998, ApJ, 502, 253

Wojdowski, P. S., Clark, G. W., \& Kallman, T. R. 2000, ApJ, 541, 963

Woo, J. W., Clark, G. W., Blondin, J. M., Kallman, T. R., \& Nagase, F. 1995, ApJ, 445, 896

Woo, J. W., Clark, G. W., Levine, A. M., Corbet, R. H. D., \& Nagase, F. 1996, ApJ, 467, 811

Yokogawa, J., Imanishi, K., Tsujimoto, M., Koyama, K., \& Nishiuchi, M. 2003, PASJ, 55, 161

Yokogawa, J., Paul, B., Ozaki, M., Nagase, F., Chakrabarty, D., \& Takeshima, T. 2000, ApJ, 539, 191 